\title{
Stability and evolution of two opposite-signed quasi-geostrophic shallow-water vortex patches
}

\author{
M.M. Jalali†, \& D.G. Dritschel †* \\ (Received 00 Month 20xx; final version received 00 Month 20xx)
}

\begin{abstract}
We examine the equilibrium forms, linear stability and nonlinear evolution of two patches having oppositesigned, uniform potential vorticity anomalies in a single-layer shallow-water flow, under the quasi-geostrophic approximation. We widely vary the vortex area ratio, the potential vorticity anomaly ratio, as well as the Rossby deformation length to unravel the full complexity of possible interactions in this system. Oppositesigned vortex interactions turn out to be far richer than their like-signed counterparts, comprehensively examined in a previous study (Jalali and Dritschel 2018, Geophys. Astrophys. Fluid Dyn. 2018, 112, 375). Unstable equilibria may evolve into a myriad of forms, many unsteady and aperiodic, and the original two vortex patches may break up into many patches which survive for long times, perhaps indefinitely.
\end{abstract}

Keywords: Quasi-geostrophy, vortex patches, contour dynamics

\section{Introduction}

Vortex dynamics continues to be a highly-researched subject which is both mathematically rich and physically relevant, particularly in geophysical fluid dynamics, which displays countless examples from ocean eddies and atmospheric mid-latitude cyclones to Jupiter's Great Red Spot and its intriguing polar vortex arrays (Adriani et al. 2018). Vortices arise spontaneously in these flows, which are characterised by enormous Reynolds numbers. Their remarkable longevity is largely due to the stabilising influences of (planetary) rotation and stratification, which together act to suppress vertical motions and vortex stretching, rendering observed flows layerwise two-dimensional (see e.g. Vallis 2006).

While vortices having a great variety of forms widely populate these natural flows, their interactions are even more varied. A commonly studied form of interaction is the merger of like-signed vortices, those having the same sense of rotation. This situation has received much attention, going back to the pioneering study of Christiansen and Zabusky (1973). Early studies focused on symmetric (identical) vortices, often vortex patches, but asymmetric vortices were found to interact in much more complicated ways, with complete merger being a relatively rare outcome (Dritschel and Waugh 1992). A review of the literature on asymmetric like-signed vortices can be found in Jalali and Dritschel (2018).

The purpose of the present work is to analyse asymmetric opposite-signed vortex interactions. Such interactions are especially commonplace in the world's oceans, which are carpeted by vortices having a very wide range of sizes (of the order of the Rossby deformation length $L_{D} \approx 10-100 \mathrm{~km}$ and smaller; see Fedorov and Ginsburg 1989, Mied et al. 1991, van Heijst and Clercx 2009). Opposite-signed vortices, called 'dipoles' widely occur and are critically important for the transport of heat, biological and chemical tracers (Carton 2010). Understanding their stability and dynamical behaviour is therefore fundamental to understanding ocean dynamics. 
To do this in any comprehensive way, a simplified approach is necessary. To this end, we consider two vortex patches having generally different areas and potential vorticity (PV) values, in a single-layer quasi-geostrophic shallow-water (QGSW) flow. Such a flow is characterised by a fundamental length scale, the Rossby deformation length $L_{D}$, arising from a competition between (background planetary) rotation and stratification (gravity acting on the free surface). Compared to the classical 2D Euler equations, the QGSW equations contain this one additional parameter, making solutions considerably richer (see e.g. Dritschel et al. 2019).

Opposite-signed vortex interactions have been studied far less extensively that like-signed ones. This is most likely because opposite-signed vortices cannot merge, and thus their interactions cannot explain the scale growth observed in two-dimensional and quasi-geostrophic turbulence (see McWilliams 1984, Larichev and McWilliams 1991, Boffetta et al. 2002, Burgess et al. 2017, Burgess and Dritschel 2019, and references). A range of equilibria in the form of translating or rotating (unbalanced) dipoles (generalising point vortices) have been computed (see e.g. Deem and Zabusky 1978, Wu et al. 1984, Dritschel 1995, Makarov and Kizner 2011, and references), but relatively few studies have examined their stability and nonlinear evolution. The first appears to be Dritschel (1995), who showed that vortex patch dipoles of generally unequal area but equal-magnitude PV generally destabilise if sufficiently close together, leading to the creation of three or more vortices at late times. The nonlinear evolution of the marginally-unstable equilibria was quantified in terms of the areas of the three largest vortices at late times, as a function of the initial area ratio. Makarov and Kizner (2011) generalised this study to unequal-magnitude PV and illustrated similar forms of destabilisation for a few selected parameter values.

To our knowledge, there has been no attempt to generalise these studies to QGSW flow, for which there is an addtional length scale $L_{D}$. The closest works are the two-layer studies of Polvani et al. (1989), Polvani (1991) and Makarov et al. (2012). None of these attempt to cover the entire (very large) parameter space, and few examples of the late-time behaviour of unstable vortex equilibria are provided. In the present work, we generalise the studies of Dritschel (1995) and Makarov and Kizner (2011) for a single-layer flow by additionally allowing for finite $L_{D}$, and by carrying out a comprehensive investigation of linear stability and nonlinear evolution. Even so, we could not be as thorough as we originally expected due to the surprising complexity of the problem. Notably, the situation is much simpler for like-signed vortex interactions for the same number of parameters (see Jalali and Dritschel 2018). In the like-signed case, the margin of stability turns out to coincide always with the first minimum in angular impulse found when decreasing the inner gap between the vortices. Moreover, the forms of nonlinear interaction may all be categorised in terms of the four fundamental forms identified by Dritschel and Waugh (1992) for equal PV vortex patches and $L_{D} \rightarrow \infty$ : (1) complete merger, (2) partial merger, (3) complete straining out and (4) partial straining out. Unfortunately, for opposite-signed vortices, no analogously simple means exists for determining linear stability, and the forms of interaction are much more varied.

The plan of the paper is as follows. In the next section, we briefly review the QGSW model and the methods used to determine equilibria and their linear stability. In section 3 , we describe the families of vortex equilibria, focusing on a few distinct cases to illustrate the surprising diversity of forms. We also discuss linear stability, and summarise how the margin of stability depends on parameters for several distinct families. In section 4 we examine the latetime nonlinear evolution for a selected set of cases. These are not meant to be representative, but rather to be indicative of the huge range of behaviour possible. No simple categorisation appears to be possible. We offer our conclusions and outlook for future research in section 5 . 


\section{Model formulation and methods}

\subsection{The quasi-geostrophic shallow water model and contour dynamics}

We consider a single-layer rotating shallow-water (SW) flow under the quasi-geostrophic (QG) approximation (see e.g. Vallis 2006, for details). The associated QGSW model is valid when the Rossby number $R o \ll 1$, i.e. when the relative vorticity $\zeta$ is small compared to the Coriolis frequency $f$, and when the Froude number $F r$ satisfies $F r^{2} \ll R o$, where $F r$ is the ratio of a typical flow speed compared with the short-scale gravity-wave speed $c=\sqrt{g H}$. Under these conditions, the free surface displacement is small compared to the mean depth $H$, and the governing equations (for an inviscid, adiabatic fluid) reduce to a single evolution equation for the potential vorticity anomaly $q$ (PV for short),

$$
\frac{\mathrm{D} q}{\mathrm{D} t}=0
$$

where $t$ is time and $\mathrm{D} / \mathrm{D} t=\partial / \partial t+\boldsymbol{u} \cdot \boldsymbol{\nabla}$ is the material derivative, along with the 'inversion relations' providing the flow field $\boldsymbol{u}$ from $q$ :

$$
\nabla^{2} \psi-\gamma^{2} \psi=q, \quad \boldsymbol{u}=\nabla^{\perp} \psi=\left(-\psi_{y}, \psi_{x}\right) .
$$

Here $\gamma=1 / L_{D}$ is the inverse Rossby deformation length and $x, y$ subscripts imply differentiation.

In this work, we consider flow in the whole $x-y$ plane and a piecewise-constant PV distribution. This enables one to formally invert (2) to find the velocity field at any point $\boldsymbol{x}$ by contour integration, i.e.

$$
\boldsymbol{u}(\boldsymbol{x})=\frac{1}{2 \pi} \sum_{k=1}^{n} \Delta q_{k} \oint_{C_{k}} K_{0}\left(\gamma\left\|\boldsymbol{x}-\boldsymbol{X}_{k}\right\|\right) \mathrm{d} \boldsymbol{X}_{k}
$$

where $\Delta q_{k}$ is the jump in $\mathrm{PV}$ across the $k$ th contour $C_{k}$, which is comprised of the points $\boldsymbol{X}_{k}$ parametrised in any convenient way. Here, $-(2 \pi)^{-1} K_{0}(\gamma r)$ is the Green function for the operator $\nabla^{2}-\gamma^{2}$, where $K_{0}$ is the modified Bessel function of the second kind, and $r$ is the distance between any two points.

The statement of PV conservation (1) implies that fluid particles either side of a contour $C_{k}$ retain their values of $\mathrm{PV}$ for all time. This implies that the flow evolution is entirely determined by the motion of the contours $C_{k}$. Thus, when $\boldsymbol{x}$ is a point on a contour, say $\boldsymbol{x}=\boldsymbol{X}_{j}^{\prime}$, we obtain a closed system of equations for the evolution of the contours

$$
\frac{\mathrm{d} \boldsymbol{X}_{j}^{\prime}}{\mathrm{d} t}=\frac{1}{2 \pi} \sum_{k=1}^{n} \Delta q_{k} \oint_{C_{k}} K_{0}\left(\gamma\left\|\boldsymbol{X}_{j}^{\prime}-\boldsymbol{X}_{k}\right\|\right) \mathrm{d} \boldsymbol{X}_{k}
$$

or "contour dynamics" (Zabusky et al. 1979, Dritschel 1989).

\subsection{Method for computing families of relative equilibria}

We consider a pair of vortex patches having different and opposite-signed values of uniform PV $q_{1}$ and $q_{2}$, and generally different areas $A_{1}$ and $A_{2}$ as depicted in figure 1 . We impose symmetry across the $y$ axis, though asymmetric equilibria are also likely to exist (LuzzattoFegiz and Williamson 2010, 2011). Such asymmetric equilibria are also likely to be generally unstable, judging from Luzzatto-Fegiz and Williamson (2010). Without loss of generality, we fix the total area $A_{1}+A_{2}=\pi$ so that the characteristic length is unity, and we also fix the total integrated PV $\Gamma=\Gamma_{1}+\Gamma_{2}=q_{1} A_{1}+q_{2} A_{2}=\pi$. Together, these give a characteristic timescale of unity. In the special case of translating equilibria where $\Gamma=0$, we take $\Gamma_{1}=\pi / 2$ and $\Gamma_{2}=-\pi / 2$. 


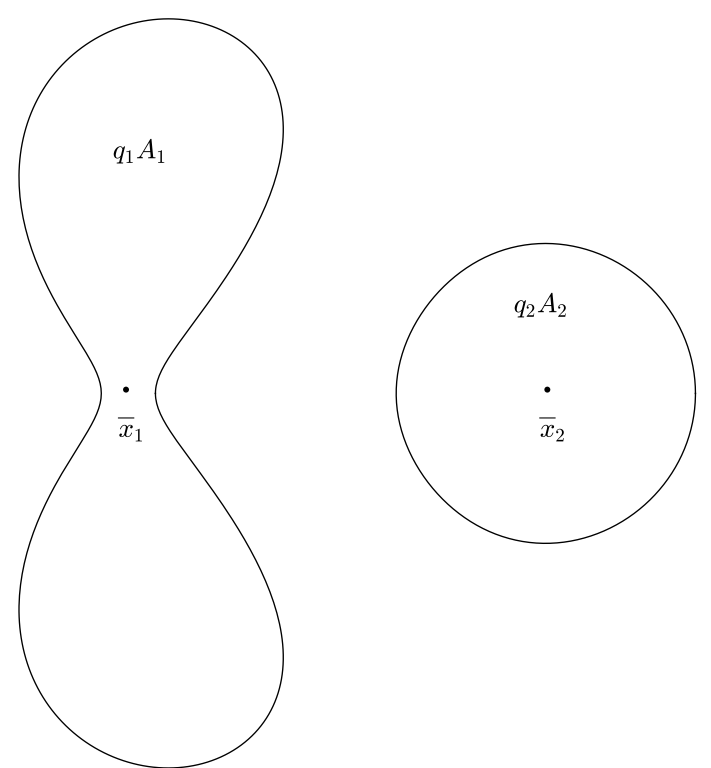

Figure 1. Schematic of a vortex patch equilibrium for two vortices having generally different areas $A_{1}$ and $A_{2}$ and different uniform values of PV $q_{1}$ and $q_{2}$ which are centered at $\bar{x}_{1}$ and $\bar{x}_{2}$.

For $\Gamma=\pi$ (rotating equilibria), it is sufficient to consider $\left|\Gamma_{2}\right|<\left|\Gamma_{1}\right|$ and all area ratios $A_{2} / A_{1}$. Defining

$$
\alpha=\frac{A_{2}}{A_{1}+A_{2}} \quad \text { and } \quad \xi=\frac{\Gamma_{2}}{\Gamma_{1}+\Gamma_{2}}
$$

all distinct equilibria have $0<\alpha<1$ and $\xi<0$. For $\Gamma=0$ (translating equilibria), all distinct equilibra have $0<\alpha \leq 1 / 2$. The patches have equal area when $\alpha=1 / 2$.

Besides the parameters $\alpha$ and $\xi$, equilibria also depend on the inverse Rossby deformation length $\gamma$ as well as the distance between the centroids of the two vortex patches, $\bar{x}_{2}-\bar{x}_{1}$, which we take to be positive without loss of generality (see figure 1).

We employ the same method as Jalali and Dritschel (2018) (see section 3.1 therein) to find families of oppositely-signed vortex equilibria, or 'relative' equilibria as they generally translate or rotate uniformly, without change of shape. This method uses numerical iteration to find new equilibria near an existing (previously computed) equilibrium, starting from an extrapolated guess when possible. Typically, entire families of equilibria are found by slowly varying a control parameter such as the outer gap between the vortices until no further solutions can be found (normally when a corner develops in one or both of the vortex patches).

Unfortunately, the solution branch so obtained can terminate prematurely, meaning that there is a fold in parameter space: two (or more) solutions may exist for the same control parameter. To navigate this difficulty, we employ the method developed in Dritschel et al. (2019), wherein either the equilibrium rotation rate $\Omega$ or the angular impulse $J$,

$$
J=\iint q\|\boldsymbol{x}\|^{2} \mathrm{~d} x \mathrm{~d} y=\frac{1}{4} \sum_{j=1}^{2} q_{j} \oint_{C_{j}}\left\|\boldsymbol{X}_{j}\right\|^{2}\left(X_{j} \mathrm{~d} Y_{j}-Y_{j} \mathrm{~d} X_{j}\right),
$$

serves as the control parameter. In the case of translating equilibria $(\Gamma=0)$, we use either the equilibrium translation rate $V$ or the ( $y$ component of) linear impulse $I$,

$$
I=-\iint q x \mathrm{~d} x \mathrm{~d} y=-\frac{1}{3} \sum_{j=1}^{2} q_{j} \oint_{C_{j}} X_{j}\left(X_{j} \mathrm{~d} Y_{j}-Y_{j} \mathrm{~d} X_{j}\right) .
$$

Each vortex patch is represented by 400 boundary points, whose spacing is adjusted to 

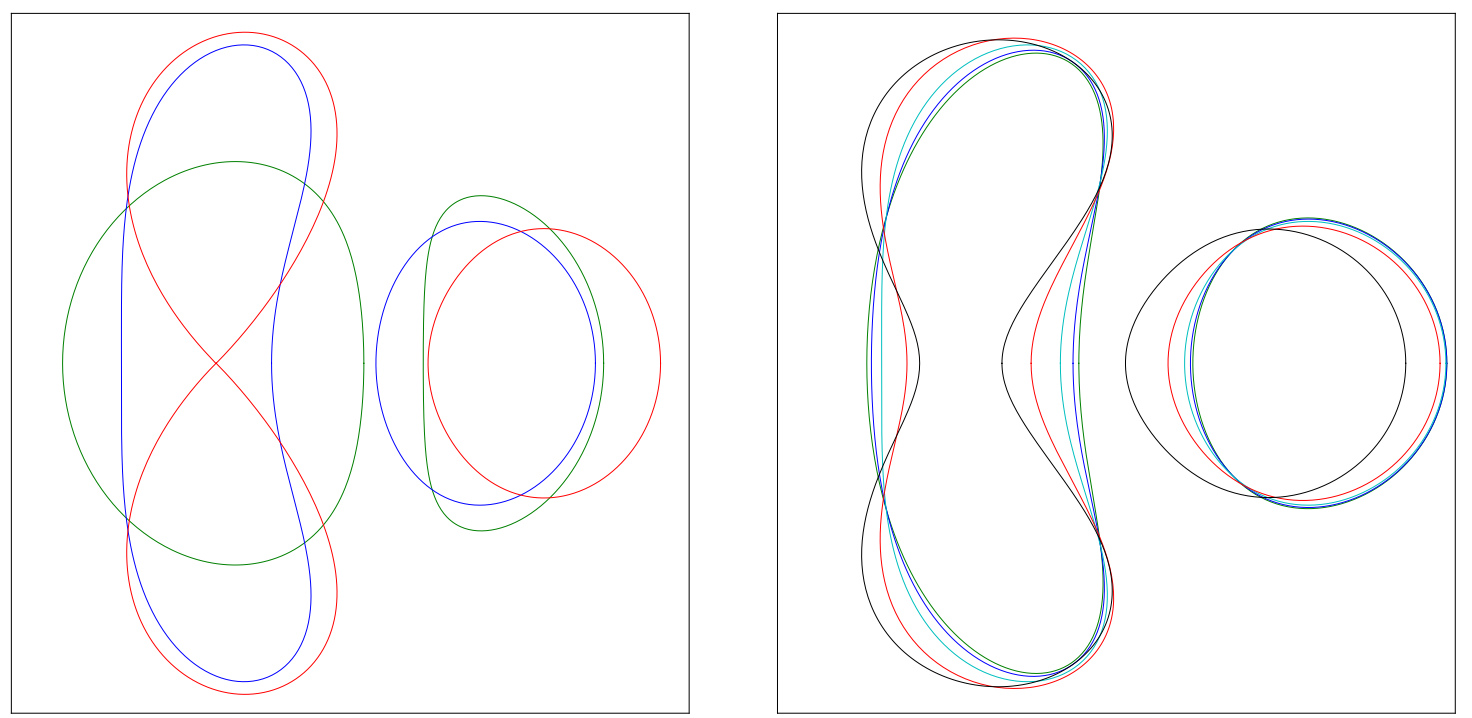

Figure 2. Equilibrium states of two vortices having $\alpha=1 / 3$ (or $\left.A_{2} / A_{1}=1 / 2\right)$ and $\xi=-1 / 3$ (or $\Gamma_{2} / \Gamma_{1}=-1 / 4$ ). Note, this implies $q_{2} / q_{1}=-1 / 2$ (vortex 1 is on the left). The left panel, for $\gamma=1$, shows 3 different states: stable ( $\Omega=0.1375891$, green), margin of stability $(\Omega=0.1149545$, blue), and last $(\Omega=0.0698399$, red). The right panel shows states at the margin of stability for different values of $\gamma$ : 0.01 (green), 0.5 (blue), 1 (cyan), 2 (red), 4 (black). (Colour online)

accurately resolve regions of high curvature. Full details can be found in Jalali and Dritschel (2018) and in Dritschel et al. (2019). We have verified that the solutions reported here are identical down to the plotted line width when 200 points are used, except in regions of relatively high curvature occurring for near limiting equilibria which tend to develop one or more corners (right angles) on their boundaries.

\subsection{Method for analysing linear stability}

We employ the same well-tested method used in a series of previous works to analyse linear stability (Dritschel 1995, Płotka and Dritschel 2013, Jalali and Dritschel 2018). The accuracy of this method is discussed extensively in Jalali and Dritschel (2018) for the closely analogous problem of like-signed QG vortex patch equilibria. Following Jalali and Dritschel (2018), we use 100 modes (sines and cosines of an appropriate travel-time coordinate) to represent disturbances (eigenfunctions) on each patch boundary. The eigenvalues, or frequencies $\sigma$ for modes evolving as $e^{-\mathrm{i} \sigma t}$, either come in pairs $\pm \sigma$ (if $\sigma$ is purely real or purely imaginary), or in quartets $\pm \sigma, \pm \sigma^{*}$, where $*$ denotes the complex conjugate, due to the underlying Hamiltonian structure of the system.

\section{Relative equilibria and linear stability}

\subsection{Rotating vortices}

We begin by illustrating a few distinct families of equilibria within the vast parameter space, starting with rotating vortices (finite $\xi$ ). The chosen families are qualitatively representative of those found more generally. The first set of families having $\alpha=1 / 3$ and $\xi=-1 / 3$ (each distinguished by $\gamma$ ) is exhibited in figure 2, with three members of the family for $\gamma=1$ shown on the left, and with members from different families $(\gamma=0.01,0.5,1,2 \& 4)$ at marginal stability on the right. For this choice of $\alpha$ and $\xi$, vortex 1 on the left deforms most strongly, and reaches a limiting 'figure of eight' state characterised by two touching right-angled corners 

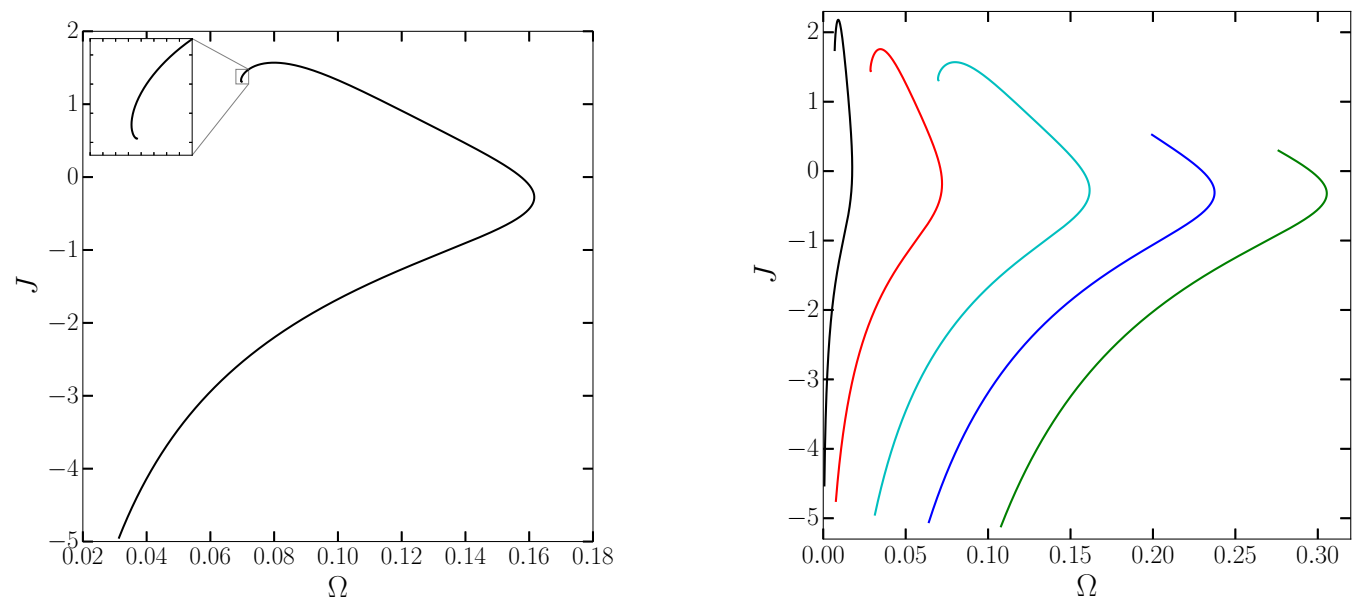

Figure 3. Left panel: angular impulse $J$ versus rotation rate $\Omega$ for the family of equilibria having $\alpha=1 / 3, \xi=-1 / 3$ and $\gamma=1$. Right panel: $J$ versus $\Omega$ for the families with (right to left) $\gamma=0.01$ (green), 0.5 (blue), 1 (cyan), 2 (red), 4 (black). (Colour online)

(note: we cannot actually compute this limiting state, but we can get very close to it; see also discussion in Dritschel et al. 2019). All states between the margin of stability and the limiting state are linearly unstable. With increasing $\gamma$, corresponding to a shortening of the interaction range, vortex 1 at marginal stability becomes progressively more deformed, while vortex 2 (which is weaker and smaller) becomes progressively more circular. The linear stability of these states is discussed in more detail below.

For values of $\alpha$ closer to but marginally less than $1 / 2$ (equal areas), progressing along a solution branch, vortex 2 on the right may begin to deform more than vortex 1 , becoming concave on the left. This then reverses near the maximum in $\Omega$, and vortex 1 becomes progressively more deformed until the limiting state. Above a critical $\alpha$, vortex 2 monitonically deforms until the limiting state is reached, as illustrated in the next case below.

Figure 3 shows the conserved angular impulse $J$ defined in (6) for the whole family of equilibria having $\alpha=1 / 3, \xi=-1 / 3$ and $\gamma=1$ as above (left panel), and for the families with different values of $\gamma$ (right panel). When the vortices are distant, $\Omega$ is relatively small and $J$ is relatively large in magnitude but negative. As the vortices come closer together, $\Omega$ reaches a maximum (for $\gamma=1$ this occurs at $\Omega=0.1615912$ ) then decreases. Further along the solution branch, $J$ reaches a positive maximum then $\Omega$ reaches a local minimum before the end of the solution branch (note: we could not reach the end of the branch for $\gamma=0.01$ and 0.5 in the right panel, though we do reach the margin of stability examined below). On the approach to the limiting state with corners, in fact the $J$ versus $\Omega$ curve likely takes the form of an infinite spiral, i.e. an infinite series of local extrema in $\Omega$ and $J$ (see Luzzatto-Fegiz and Williamson 2010). Nevertheless, these near corner states turn out to be highly unstable. With increasing $\gamma$ (see right panel), the angular velocity $\Omega$ decreases as expected while the range of $J$ remains comparable.

The linear stability of the family of equilibria having $\alpha=1 / 3, \xi=-1 / 3$ and $\gamma=1$ is examined in figure 4. Shown are the frequencies $\sigma_{r}$ in blue and the growth rates $\sigma_{i}$ in red (the right panel shows a zoomed portion of the left). The margin of stability occurs close to $\Omega=0.115$ along the portion of the solution branch where $\Omega$ is decreasing. Two opposite frequencies coalesce at the margin of stability and split into a growing and decaying mode with zero frequency for smaller $\Omega$. These frequencies lie on anti-symmetric curves which fold over at $\Omega=0.1615912$ and continue to grow in magnitude as one moves backwards along the solution branch (they limit to one of the natural frequencies existing on circular vortex patches when the patches are infinitely distant). Moving forward along the solution branch 

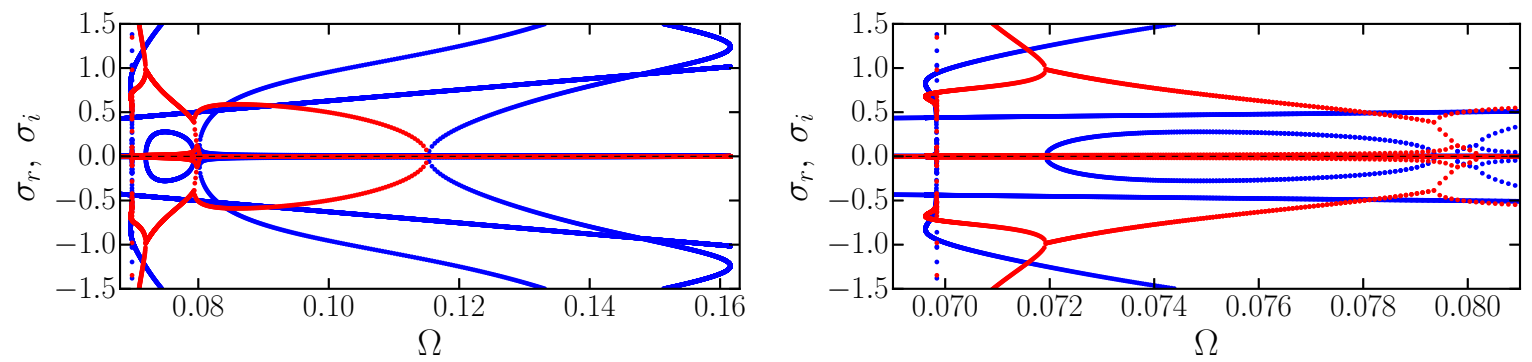

Figure 4. Left panel: frequencies $\sigma_{r}$ (blue) and growth rates $\sigma_{i}$ (red) as a function of rotation rate $\Omega$ for two vortices having $\alpha=1 / 3, \xi=-1 / 3$ and $\gamma=1$. Right panel: zoom showing the range $0.069 \leq \Omega \leq 0.081$. Note that $\sigma_{r}$ and $\sigma_{i}$ are multi-valued functions of $\Omega$ due to the turning points at both ends of the range in $\Omega$. (There are many other higher frequency modes not visible in the domain of view; these are all stable modes.) (Colour online)

past the margin of stability (decreasing $\Omega$ ), a second pair of opposite frequencies coalesces around $\Omega=0.0800$ (see right panel), briefly giving rise to a growing-decaying mode pair for smaller $\Omega$. Shortly thereafter at around $\Omega=0.0794$, these growing and decaying modes coalesce with the original growing and decaying modes erupting at $\Omega=0.115$, and acquire non-zero frequencies (see the blue 'loop' between $\Omega=0.072$ and $\Omega=0.0794$ approximately). Around $\Omega=0.072$, these opposite frequencies coalesce, the weaker modes disappear, and the stronger modes (all having zero frequency) split into a weaker and stronger pair. The stronger modes here are expected to diverge, i.e. $\left|\sigma_{i}\right| \rightarrow \infty$ as one approaches the limiting corner state, since at a corner velocity derivatives diverge (Overman II 1986, Dritschel et al. 2019).

In the case of like-signed vortex patches examined in Jalali and Dritschel (2018), the margin of stability coincides with the first extremum in $J$ encountered along the solution branch emanating from infinitely distant vortices. For opposite-signed vortex patches, by contrast, this does not always occur. For the family examined in figure 4, marginal stability is found near $\Omega=0.115$, well before $J$ reaches a maximum close to $\Omega=0.0800$. At this point, a new instability erupts, but it is not the first one encountered along the solution branch. Both instabilities are 'exchange type', where a pair of opposite frequencies coalesce and give birth to a pair of opposite growth rates. Even the new instability appearing at $\Omega=0.072$ involves the coalescence of opposite frequencies, though in this case modes with non-zero growth rates bifurcate into pairs.

We next contrast the set of families having $\alpha=1 / 3$ and $\xi=-1 / 3$, just described, with the set having $\alpha=3 / 5$ and $\xi=-2 / 5$. A few representative vortex equilibria are shown in figure 5, with $\gamma=1$ on the left and marginally-unstable states for various $\gamma$ on the right. Now the vortex on the right (vortex 2) is more deformed, and has a limiting state consisting of two lobes connected at a single point. This vortex is weaker both in terms of PV $q$ and integrated PV $\Gamma$, though larger in area $A$. Notably, which vortex deforms the most is not simply determined by these considerations (in the previous set of families, the stronger vortex deforms the most); see also Jalali and Dritschel (2018).

The dependence of the angular impulse $J$ on $\Omega$ along the solution branches is shown in figure 6. Compared with the previous set of families shown in figure 3 , the general form of the curves is significantly different. First, for $\gamma=1$ (left panel), $J$ increases with $\Omega$ until just beyond the turning point at $\Omega=\Omega_{\max }=0.09515$, then decreases with $\Omega$ until reaching another turning point near the limiting state having $\Omega \approx 0.058115$ (see the insert). This is likely the start of an infinite spiral toward the limiting state. Another distinct feature for this set of families is that $J<0$ for all states. The effect of increasing $\gamma$ is mainly to reduce $\Omega$, as found previously.

The frequencies and growth rates for the family with $\alpha=3 / 5, \xi=-2 / 5$ and $\gamma=1$ are shown in figure 7 . Compared to the previous family in figure 4 , the situation is now 

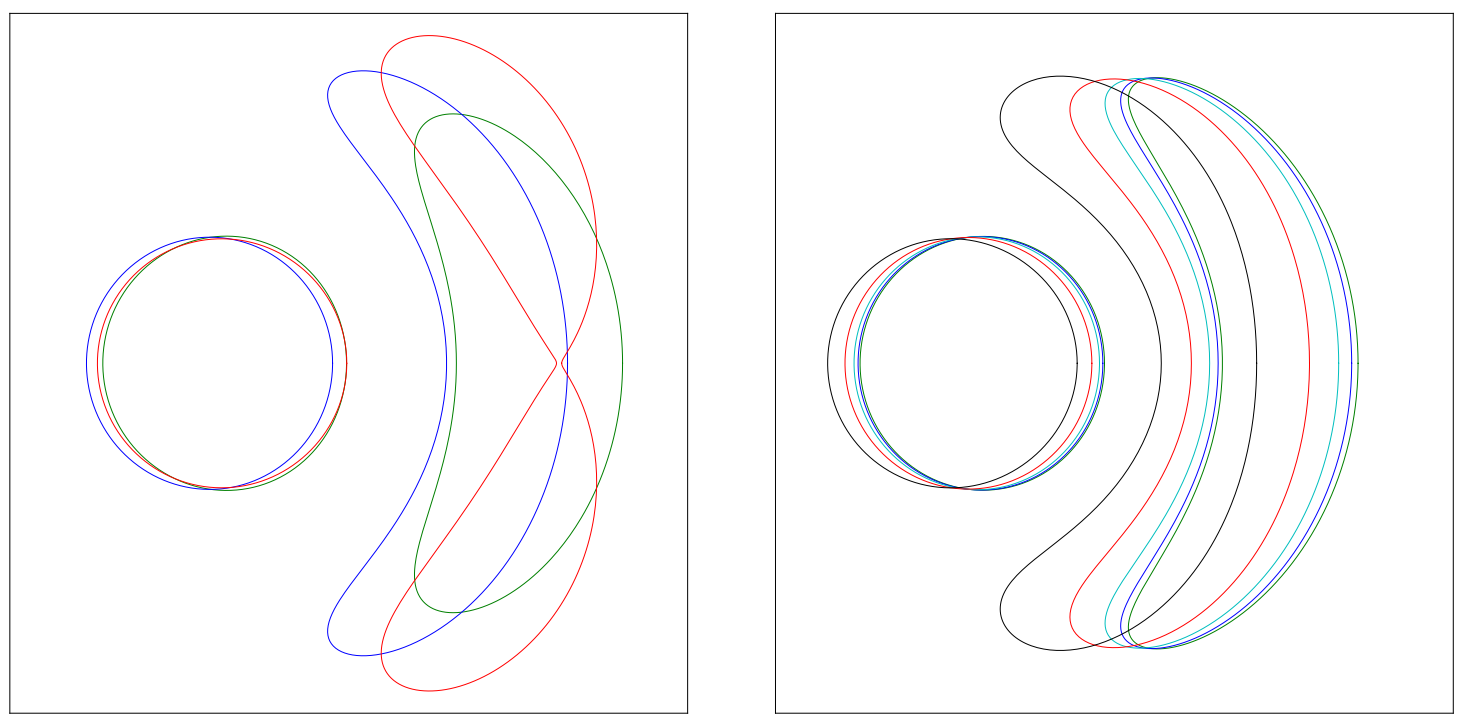

Figure 5. Equilibrium states of two vortices having $\alpha=3 / 5$ (or $\left.A_{2} / A_{1}=3 / 2\right)$ and $\xi=-2 / 5$ (or $\Gamma_{2} / \Gamma_{1}=-2 / 7$ ). Note, this implies $q_{2} / q_{1}=-4 / 21$ (vortex 1 is on the left). The left panel, for $\gamma=1$, shows 3 different states: stable ( $\Omega=0.0815425$, green), margin of stability $(\Omega=0.0944402$, blue), and last $(\Omega=0.0581150$, red). The right panel shows states at the margin of stability for different values of $\gamma$ : 0.01 (green), 0.5 (blue), 1 (cyan), 2 (red), 4 (black). (Colour online)
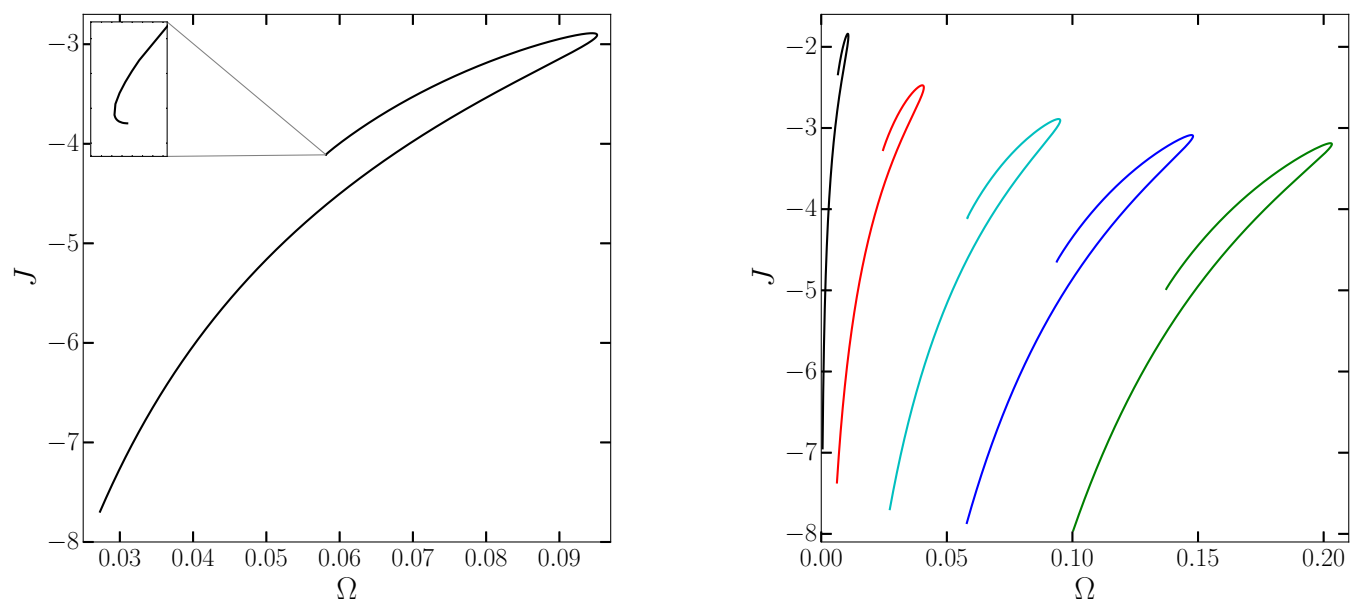

Figure 6. Left panel: angular impulse $J$ versus rotation rate $\Omega$ for the family of equilibria having $\alpha=3 / 5, \xi=-2 / 5$ and $\gamma=1$. Right panel: $J$ versus $\Omega$ for the families with (right to left) $\gamma=0.01$ (green), 0.5 (blue), 1 (cyan), 2 (red), 4 (black). (Colour online)

simpler. Just beyond the turning point at $\Omega=0.09515$, two opposite frequencies coalesce and lead to the emergence of a stable and unstable mode with opposite growth rates around $\Omega=0.09445$, and these modes remain dominant along the remainder of the solution branch, with $\left|\sigma_{i}\right|$ showing divergent behaviour on the approach to the limiting corner solution. A second weaker instability erupts from another coalescence of opposite frequencies around $\Omega=0.0735$ and exists up to the limiting corner solution. Both sets of stable and unstable modes have zero frequency. Notably, the point of marginal stability for this family coincides with the first maximum of $J$, which occurs at $\Omega=0.09445$ (see figure 6 ). This is in contrast with the previous family, $\alpha=1 / 3, \xi=-1 / 3$ and $\gamma=1$, where the point of marginal stability occurs well before the first maximum in $J$ (see figures 3 and 4). In that family, the first maximum in $J$ coincides with a secondary instability, at smaller $\Omega$ further along the solution branch. 


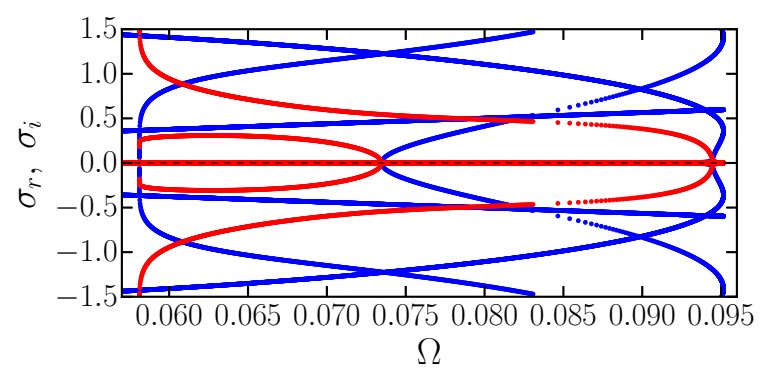

Figure 7. Frequencies $\sigma_{r}$ (blue) and growth rates $\sigma_{i}$ (red) as a function of rotation rate $\Omega$ for two vortices having $\alpha=3 / 5, \xi=-2 / 5$ and $\gamma=1$. Note that $\sigma_{r}$ and $\sigma_{i}$ are multi-valued functions of $\Omega$ due to the turning points at both ends of the range in $\Omega$. (There are many other higher frequency modes not visible in the domain of view; these are all stable modes.) (Colour online)
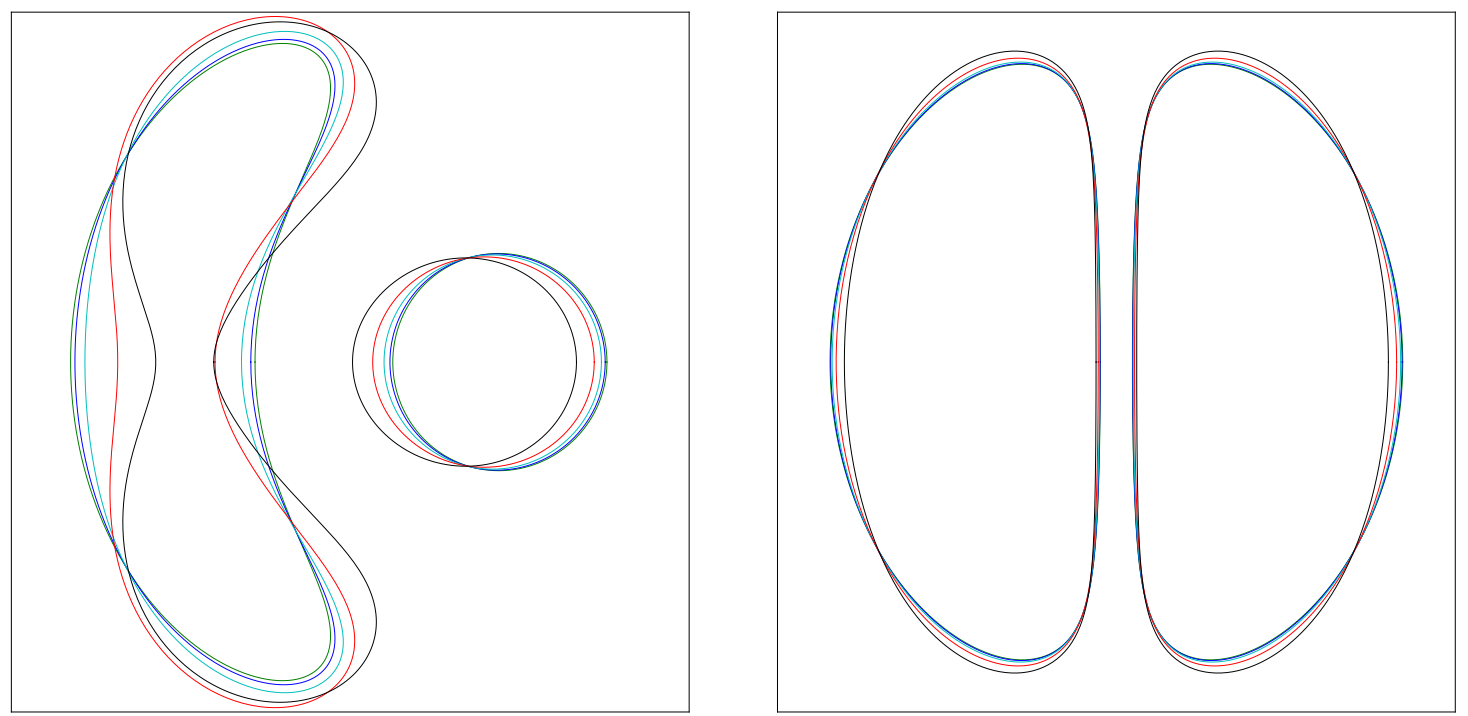

Figure 8. Equilibrium states of two translating vortices $(\xi \rightarrow-\infty)$ at marginal stability, with $\alpha=1 / 4$ (i.e. $A_{2} / A_{1}=1 / 3$ $\& q_{2} / q_{1}=-3$ ) on the left and $\alpha=1 / 2$ (i.e. $A_{2} / A_{1}=1 \& q_{2} / q_{1}=-1$ ) on the right. The different states shown are for different values of $\gamma: 0.01$ (green), 0.5 (blue), 1 (cyan), 2 (red), 4 (black). (Colour online)

\section{2. $\quad$ Translating vortices}

We next turn to the situation when the total integrated PV is zero, i.e. translating vortices. Two sets of families are contrasted in figure 8 , one which is asymmetric $(\alpha=1 / 4)$ on the left, and the other which is symmetric $(\alpha=1 / 2)$ on the right. In the latter, the vortex patches have equal areas but opposite values of PV. Their shapes are strikingly different from their asymmetric counterparts, and moreover they hardly change with $\gamma$ over a considerable range. By contrast, the asymmetric vortex shapes do vary significantly with $\gamma$, and are qualitatively similar to those found for rotating equilibria in figures $2 \& 5$. Notably, a small difference in vortex areas is enough to lead to a major asymmetry in the vortex shapes at marginal stability and beyond. That is, symmetric vortices are exceptional.

The dependence of linear impulse $I$ on translation speed $V$ for these families of equilibria and for other families having different $\gamma$ is shown in figure 9 , with $\alpha=1 / 4$ on the left and $\alpha=1 / 2$ on the right. The asymmetric equilibria (left) all show a turning point in $V$, followed by a minimum in $I$, then an increase in $I$ with decreasing $V$. Approaching the limiting corner state, the $I$ versus $V$ curve begins to form a spiral, as found for the rotating families of equilibria above.

By contrast, the symmetric equilibria show an apparently monotonic dependence of $I$ on $V$, 

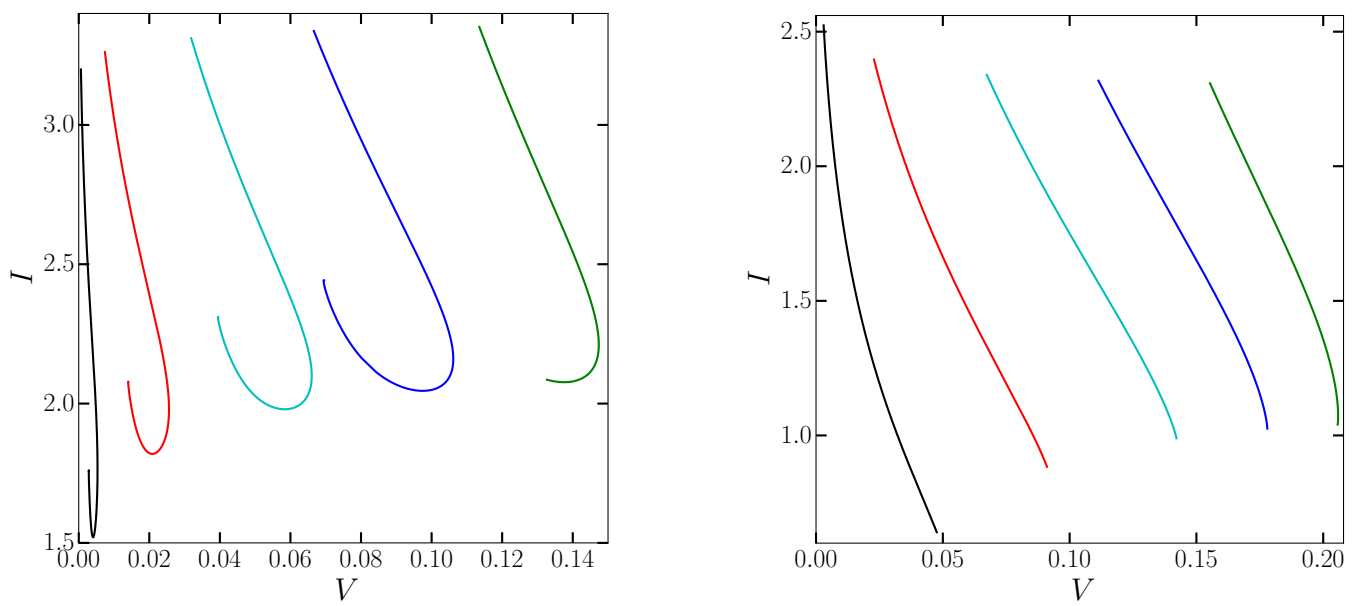

Figure 9. Left panel: linear impulse $I$ versus translation speed $V$ for the families of translating equilibria having $\alpha=1 / 4$ right panel: same but for $\alpha=1 / 2$; in each panel (right to left) $\gamma=0.01$ (green), 0.5 (blue), 1 (cyan), 2 (red), 4 (black) (Colour online)

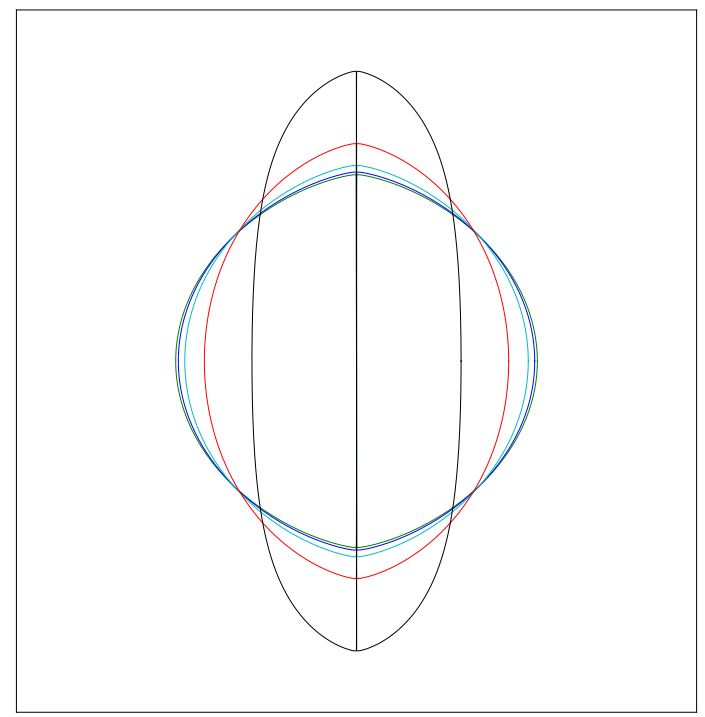

Figure 10. Near limiting equilibrium states of two symmetric translating vortices $(\alpha=1 / 2)$ for different values of $\gamma$ : 0.01 (green), 0.5 (blue), 1 (cyan), 2 (red), 4 (black). (Colour online)

though in fact the solution branch for $\gamma=0.01$ (shown in green) has a turning point near its end. No turning point is found for the other values of $\gamma$ considered, despite the fact that all of the near limiting states have incipient corners on their boundaries - see figure 10. Note, for $\gamma \leq 0.5$, the contour shapes are almost identical, but as $\gamma$ becomes large, the limiting states elongate and acquire a nearly uniform thickness in $x$ (proportional to $L_{D}=\gamma^{-1}$ ) except near the $y$ extremities.

The linear stability of the asymmetric translating equilibia is qualitatively similar to that found for the rotating equilibria in figure 7 , with the margin of stability occuring at the first minimum in $I$, here at $I=0.0584$ for $\gamma=1$, then a secondary instability occurring further along the branch at $I=0.0482$. The first instability is 'exchange type', where a pair of opposite frequencies coalesce and give rise to a pair of oppositely growing modes with zero frequency. The picture simplifies for larger $\gamma$, with the first instability remaining dominant for all $V$ beyond the margin of stability (not shown).

By contrast, the margin of stability for symmetric translating equilibia does not coincide 


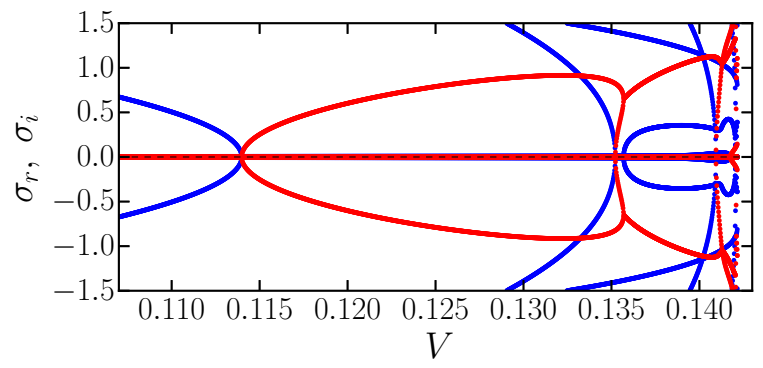

Figure 11. Frequencies $\sigma_{r}$ (blue) and growth rates $\sigma_{i}$ (red) as a function of translation rate $V$ for two symmetrical translating vortices having $\alpha=1 / 2$ and $\gamma=1$. (Colour online)

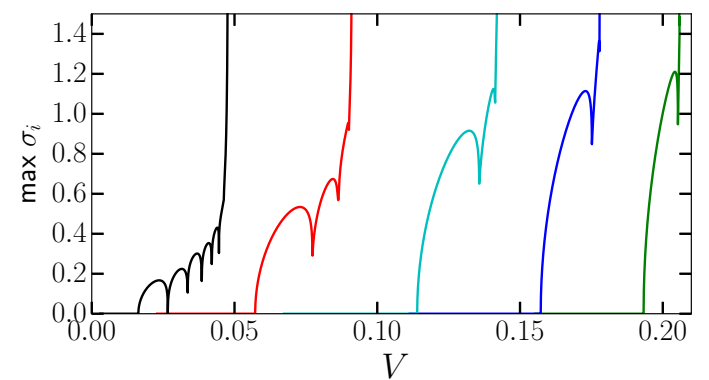

Figure 12. Maximum growth rate versus translation rate $V$ for two symmetrical translating vortices, $\alpha=1 / 2$, for (right to left) $\gamma=0.01$ (green), 0.5 (blue), 1 (cyan), 2 (red) and 4 (black). (Colour online)

with a minimum in $I$. However, it is an 'exchange-type' instability in which a pair of modes having opposite frequencies coalesce and give rise to a pair of modes having opposite growth rates, see figure 11. Moreover, the eigenfunction is asymmetric, implying that a new branch of asymmetric solutions erupts from the margin of stability. This can be deduced by searching for slightly asymmetric equilibria ('imperfect equilibria', see Luzzatto-Fegiz and Williamson 2010, 2011, Dritschel et al. 2019), which follow the symmetric branch up to the margin of stability and then rapidly diverge from it. We have only been able to follow a short section of this asymmetric branch before our numerical methods diverge, possibly as a result of further instabilities. Nonetheless, this and any subsequent branches are generally unstable (Dritschel et al. 2019). Finally, as $\gamma$ increases, a progression of instabilities arises due to the elongation of the equilibrium states - see figure 12 .

\subsection{Equilibrium properties at marginal stability}

We next consider several distinct values of the integrated PV fraction $\xi=\Gamma_{2} /\left(\Gamma_{1}+\Gamma_{2}\right)$ and examine the dependence of the equilibrium properties at marginal stability on the area fraction $\alpha=A_{2} /\left(A_{1}+A_{2}\right)$ and the inverse Rossby deformation radius $\gamma$. This analysis provides a clearer view of the wider parameter space, but is by no means comprehensive. Nonetheless, the patterns observed are typical of many other values of $\xi$ we have explored.

Figure 13 summarises the dependence of $J$ and $\Omega$ on $\alpha$ at marginal stability, for three different integrated PV ratios $\xi$. The most striking feature is the discontinuity near $\alpha=0.5$. This is related to which vortex is more deformed; at smaller $\alpha$, vortex 1 (on the left) is more deformed as in figure 2(a), while at larger $\alpha$, vortex 2 is more deformed as in figure 5(a). To the right of the discontinuity, in all cases the margin of stability coincides with the first maximum in $J$, whereas to the left, the margin of stability occurs before this point along the solution branch. 

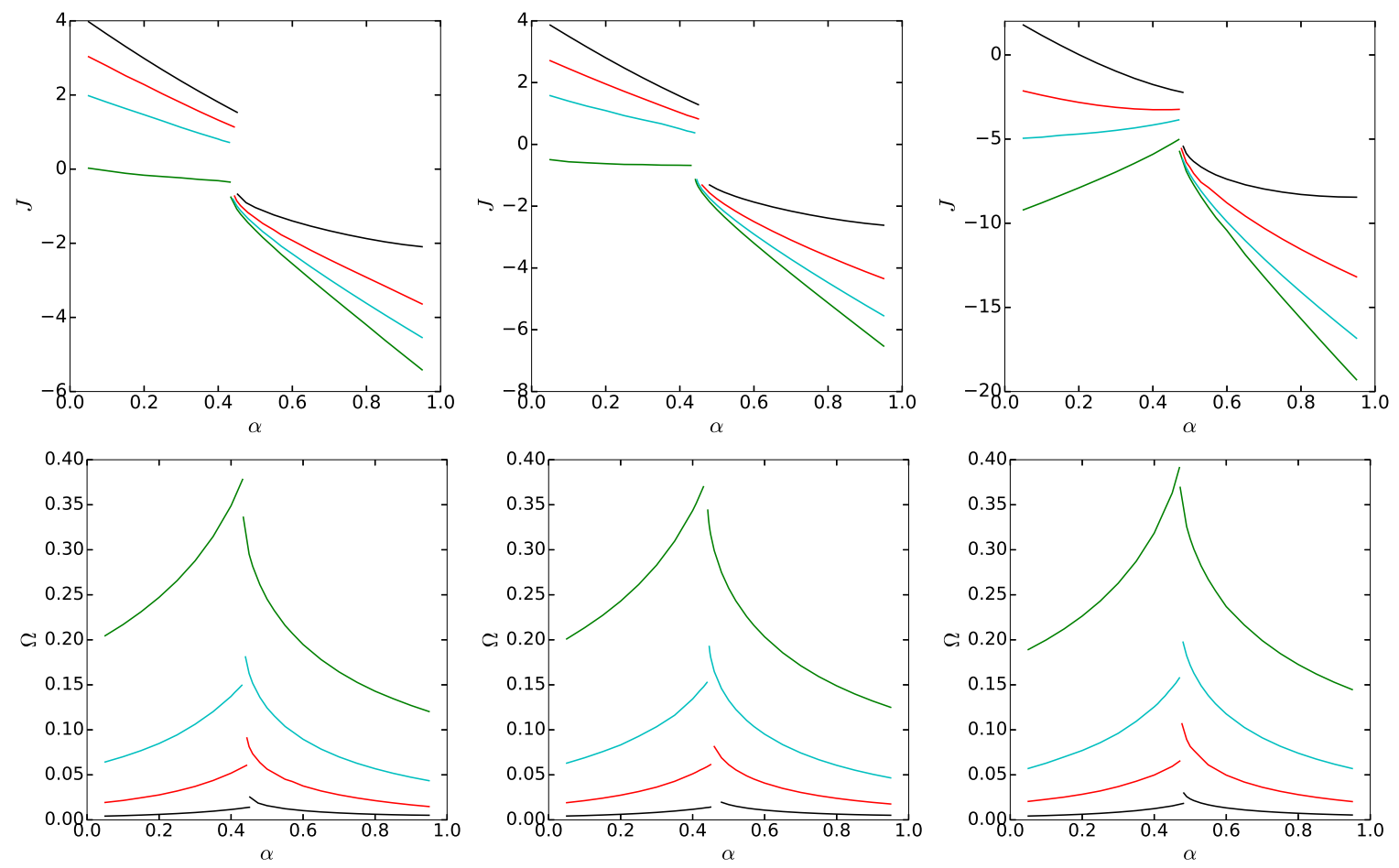

Figure 13. Angular impulse $J$ (top row) and rotation rate $\Omega$ (bottom row) versus area ratio $\alpha$ at marginal stability for $\xi=-1 / 3$ (left), $\xi=-2 / 5$ (middle) and $\xi=-1$ (right). Each panel shows four values of the inverse Rossby deformation length: $\gamma=0.01$ (green), $\gamma=1$ (cyan), $\gamma=2$ (red) and $\gamma=4$ (black). (Colour online)
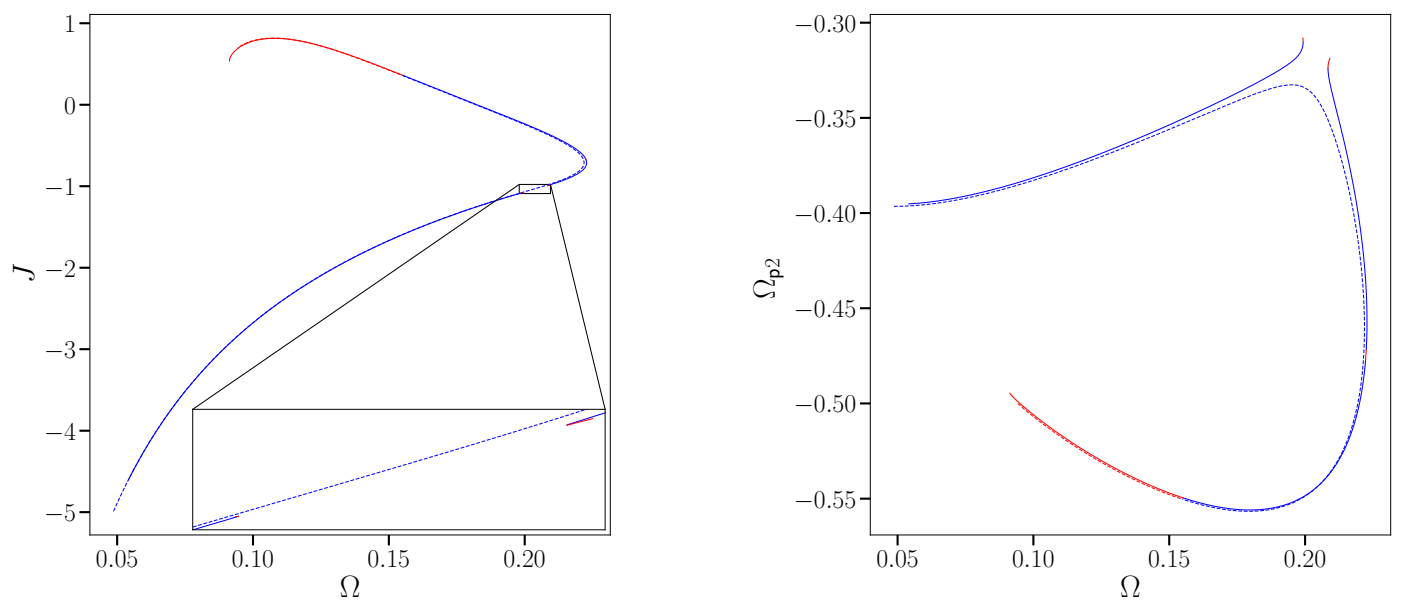

Figure 14. Left panel: angular impulse $J$ versus rotation rate $\Omega$ for two families of equilibria having $\xi=-0.4, \gamma=1$ and slightly different values of $\alpha$ : 0.444 (dashed lines) and 0.445 (solid lines). The blue portions of the curves are linearly stable, while the red are linearly unstable (see insert for detail). Right panel: same except for the particle frequency $\Omega_{\mathrm{p} 2}$ of vortex 2 . The later is defined as $2 \pi$ divided by the rotation period of a fluid particle around the boundary of vortex 2. (Colour online)

The discontinuity implies that there is a critical value of $\alpha$ (for a given choice of $\xi$ and $\gamma$ ) for which the solution branch bifurcates at some point into two sub-branches (for $\xi=-0.4$ and $\gamma=1$, the critical value of $\alpha$ lies between 0.444 and 0.445$)$. The branches lead to limiting states where either vortex 1 or vortex 2 is deformed into a near figure of 8 . A similar situation arises for a singly-connected vortex patch in Dritschel et al. (2019). It implies that there exists separated branches of solutions for other values of $\alpha$ (at least near enough to the discontinuity). 

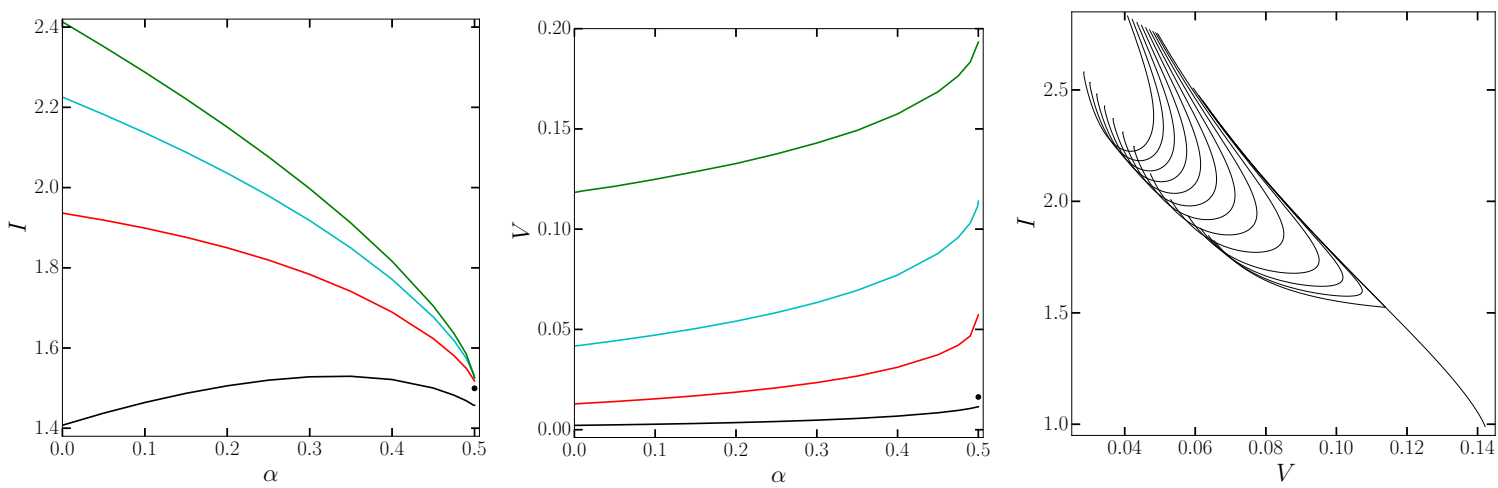

Figure 15. Linear impulse $I$ (left) and translation speed $V$ (middle) versus area ratio $\alpha$ at marginal stability for translating equilibria with (top to bottom) $\gamma=0.01$ (green), 1 (cyan), 2 (red) and 4 (black). The separate black dot is for $\gamma=4$ and $\alpha=0.5$ identically (see text for explanation). The right panel shows $I$ versus $V$ when $\gamma=1$ for a range of $\alpha: 0.001,0.05,0.1,0.15,0.2,0.25,0.3,0.35,0.4,0.45,0.475,0.49,0.49999$ and 0.05 (left to right starting from the top left). The curve for $\alpha=0.5$ is the only one without a turning point. (Colour online)

This is shown explicitly in figure 14 for the families of solutions having $\alpha=0.444$ and 0.445 mentioned above. The single solution branch for $\alpha=0.444$ (dashed curve) breaks into two branches for $\alpha=0.445$. The upper branch in the left panel (corresponding to the lower branch in the right panel) separates from the main branch coming from infinitely distant vortices (many other examples of these are given in Dritschel et al. 2019). The branch bifurcation is most clearly seen in the behaviour of the particle frequency $\Omega_{\mathrm{p} 2}$ of vortex 2 . At the left end of this separated branch, vortex 1 limits to an unstable figure of 8 shape, while at the other, the vortices likely limit to a state with corners near their extremities in $y$, as occurs for symmetric translating vortices (see figure 10). However, we were unable to compute the rest of this branch, though it appears to be unstable. Also, the main branch likely continues to a limiting state with vortex 2 exhibiting a figure of 8 shape as in figure 5 (a), but again we were unable to follow this branch all the way there. Note that the branch bifurcation leads to two additional margins of stability, one of which continues the curves to the left of the discontinuity in Figure 13 to larger $\alpha$, while the other folds back the curves to the right of the discontinuity, making them multi-valued. We have not attempted to generate the complete curves due to the difficulty in finding these separated solution branches. Both portions of these separated branches are unstable, seen most clearly at the tips of the curves in the upper right part of the right panel.

Returning to figure 13, as $\xi$ increases in magnitude (left to right), the range in $J$ expands, and the dependence on $\alpha$ (for $\alpha<0.5$ ) changes. For $\xi=-1$ (right), $J$ no longer monotonically decreases for the smaller value of $\gamma=0.01$. On the other hand, $\Omega$ in all cases first increases with $\alpha$, peaks near $\alpha=0.5$ (equal area vortices), then subsequently decreases. The discontinuity in $\alpha$ shifts to slightly larger values as $|\xi|$ increases but remains less than 0.5 .

As $\gamma$ increases, $J$ increases, consistent with the increase in distortion of the vortices as seen for example in figure $2(\mathrm{~b})$. On the other hand, $\Omega$ decreases strongly due to the shortening of the interaction range.

Figure 15 provides the same summary for translating vortices at marginal stability, with the dependence of linear impulse $I$ on $\alpha$ shown on the left, and the dependence of the translation speed $V$ on $\alpha$ shown in the middle; the additional panel on the right shown $I$ versus $V$ only for $\gamma=1$ but for a wide range of $\alpha$ values, as indicated. Note, only $\alpha \leq 0.5$ needs to be considered since the equilibria for $\alpha>0.5$ are mirror symmetric across the $y$ axis. Regarding $I$, this property generally decreases with $\alpha$ except when $\gamma$ is large; only $\gamma=4$ shows a nonmonotonic behaviour of $I$ on $\alpha$. As $\alpha \rightarrow 0.5$, the values of $I$ become closely similar, consistent with the similar vortex shapes found in figure $8(\mathrm{~b})$. Notably, there are no discontinuities like 
those occurring for rotating vortices, except in the case $\gamma=4$ at $\alpha=0.5$ (see below). Moreover, $I$ decreases as $\gamma$ increases, consistent with the definition of $I$ in $(7)$ and the observation that the vortices occupy a smaller range in $x$ as seen for example in figure 8(a). The dependence of $V$ on $\alpha$ is simple, increasing slightly with $\alpha$. The strong variation with $\gamma$ reflects the decreasing strength of interaction with increasing $\gamma$, as expected.

Apart from the symmetric equilibria having $\alpha=0.5$, the margin of stability always coincides with the first minimim in $I$. For symmetric equilibria, the branches with $\gamma>0.01$ do not have a minimum in $I$ before the limiting state. The margin of stability in these cases (and also in the case $\gamma=0.01$ ) coincides with a branch bifurcation where a new asymmetric sub-branch emerges. This is shown explicitly for $\gamma=1$ in the right panel of figure 15 . The solution branch for $\alpha=0.49999$ appears to bifurcate from the branch for $\alpha=0.5$ near $V=0.113946$ and $I=1.52486$. This is precisely where the margin of stability occurs when $\alpha=0.5$, showing that the instability is associated with the emergence of an unstable sub-branch.

When $\gamma=4$ (and presumably larger), the sub-branch which emerges at the margin of stability for $\alpha=0.5$ is in fact stable until it reaches a minimum in $I$. This has been verified by computing the family of solutions for $\alpha=0.49999$, which tracks along the main $\alpha=0.5$ branch until its margin of stability, then moves away and downwards (towards decreasing $I$ ) until it reaches a minimum where it becomes unstable (not shown). This is unlike in the case $\gamma=1$ discussed above, where the bifurcating sub-branch moves upwards in $I$, so its minimum coincides with the margin of stability. The same is true for $\gamma=0.01$ and $\gamma=2$. Only $\gamma=4$ shows anomalous behaviour.

\section{Nonlinear evolution}

We next examine a selection of unstable equilibria to explore the possible forms of nonlinear evolution and determine the nature of the late-time flow states. Over 200 simulations have been performed but we focus on only a few to give a hint at the great diversity of behaviour.

Simulations were performed using 'Contour Surgery' (Dritschel 1988, 1989), an extension of 'Contour Dynamics' (Zabusky et al. 1979) permitting topological reconnections (splitting, merger, etc). We use the standardised settings for the numerical parameters given in Fontane and Dritschel (2009), namely a dimensionless node separation of $\mu=0.2$, a large-scale length $L=\pi / 40$ and a time step $\Delta t=0.02$. The choice of $L$ ensures that approximately 400 nodes are used around a circular contour of unit radius (the same number used on each equilibrium vortex patch) - a relatively high resolution compared to many past studies. The choice of $\Delta t$ ensures accurate resolution in time, for the range of $\mathrm{PV}$ values considered. Note: in the simulations conducted, $q_{1}$ and $q_{2}$ are multiplied by a factor of $2 \pi$ so that one unit of time is comparable to a vortex turn-around time (for $\gamma \ll 1$ ). For rotating states this means $q_{1}=2 \pi(1-\xi) /(1-\alpha)$ and $q_{2}=2 \pi \xi / \alpha$, while for translating states this means $q_{1}=\pi /(1-\alpha)$ and $q_{2}=-\pi / \alpha$.

While numerical errors are inevitable and, in particular, affect detailed aspects of the longtime dynamics, every effort was made to conduct the most accurate simulations affordable. Typical simulations required days of computing time on an Intel i7-8700 (single processor) clocked at $3.2 \mathrm{GHz}$. Hundreds of simulations were carried out, representing well over a year of computer time.

\subsection{Rotating vortices}

We start by exploring the family of solutions for $\alpha=1 / 3, \xi=-1 / 3$ and $\gamma=1$ featured in figure 2(a). As a guide, figure 16 shows the maximum growth rate $\sigma_{i}$ versus $\Omega$ for this family, along with four points where we have performed simulations of the nonlinear evolution. For 


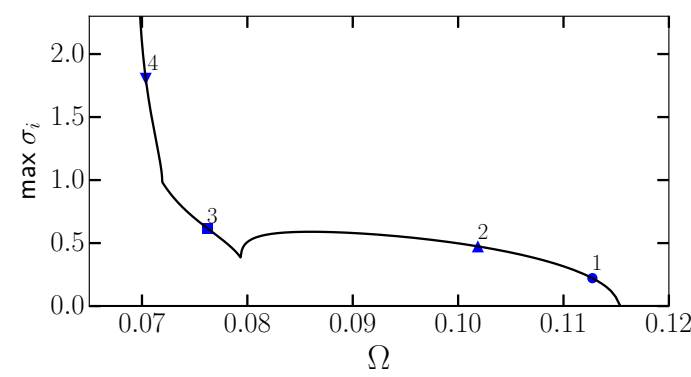

Figure 16. Maximum growth rate versus rotation rate $\Omega$ for two vortices having $\alpha=1 / 3, \xi=-1 / 3$ and $\gamma=1$. The numbered points mark different simulations discussed below. (Colour online)

more detail, please also consult figure 4 , which shows $\sigma_{i}$ and $\sigma_{r}$ for all modes.

Starting with point 1 near the margin of stability, referred to as 'case 1', figure 17 shows a few selected times from the flow evolution (see also supplementary movie 1). In this and subsequent simulations, the instabilities grow purely from numerical 'noise', i.e. from imperfections in the contour shapes and the finite accuracy of the numerical method. The evolution is shown in a frame of reference rotating with the equilibrium state and centred at the origin. First of all, the instability first pinches off a lobe from the larger vortex on the left around $t=40$, which is ejected behind the vortices and pairs with another lobe torn off the smaller original vortex between $t=40$ and 45 . The two lobes end up in the lower left at $t=45$, with the lobe torn off the smaller vortex being the more distorted of the two. The various filaments of PV created as the vortices are pulled apart themselves roll up, creating a multitude of small vortices (many thousand at this resolution!). The four main vortices remain largely intact until around $t=90$ when two parts of the originally smaller vortex begin to merge. This more or less completes by $t=110$, not without considerable complexity developing on the boundary of the merged vortex. Thereafter, the flow simplifies somewhat, leaving two smaller vortices of opposite sign orbiting around the central larger vortex. The opposite-signed vortex lies between two like-signed vortices in radius. While unsteady, this configuration appears to be persistent, at least to $t=1000$ when the simulation was discontinued. While the numerics cannot be expected to accurately capture all of the fine debris despite the very high resolution employed, the final state of three vortices is likely to be a robust outcome. This case highlights the fact that opposite-signed vortex interactions generally lead to a decrease in vortex sizes, never an increase. This behaviour strongly differs from that found for like-signed vortices, which can both increase and decrease in size (Dritschel and Waugh 1992, Jalali and Dritschel 2018).

We next consider a more unstable state, case 2, further along the branch of solutions in figure 16. This case exhibits the same mode of linear instability as case 1 , as seen in more detail in figure 4. The nonlinear evolution is shown in figure 18 at a few selected times (see also supplementary movie 2). The early evolution is a little simpler than in the previous case, with the largest vortex splitting into two unequal parts and the smaller vortex escaping unscathed, but only until the dipole in the lower right corner at $t=50$ sweeps around and collides with it by $t=70$. This causes the smaller vortex to split, and one part of it ends up in the small dipole in the upper right corner at $t=75$. For the remainder of the simulation (and indeed until $t=1000$ when the simulation was discontinued), this dipole remains in a distant orbit around the largest, central vortex. The vortex occupying the inner orbit is opposite in sign to the central vortex, and originally belonged to vortex 2 on the right at $t=0$. Despite the fact that the vortices occasionally become close together as at $t=300$, they remain robust for long times. There are also many more smaller vortices circulating around and between the four main vortices.

Moving further along the solution branch to case 3 in figure 16, the mode of linear instability 


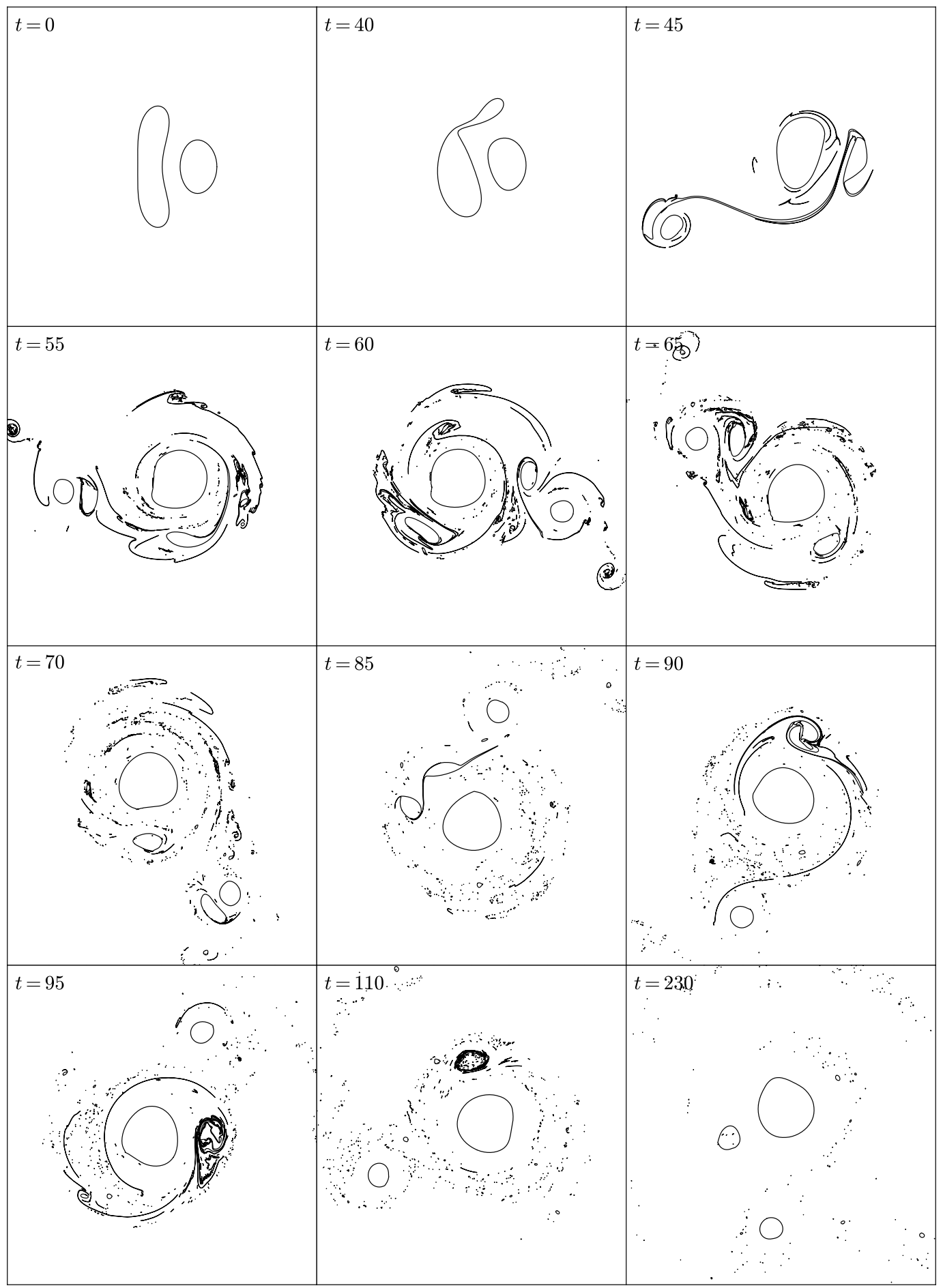

Figure 17. Case 1: nonlinear evolution from the equilibrium state having $\alpha=1 / 3, \xi=-1 / 3, \gamma=1$ and $\Omega=0.112739$. The bounding box is used purely to separate different time frames; the actual computational domain is unbounded.

changes to one with non-zero frequency (see figure 4). The growth rate is also a little larger compared to cases 1 and 2 previously discussed. The nonlinear evolution is shown in figure 19 (see also supplementary movie 3). First of all, it is much simpler than the other cases, despite the larger growth rate of the instability. The initially larger vortex splits cleanly into two nearly equal halves. Subsequently, the vortices perform a dance, with one of the two halves 


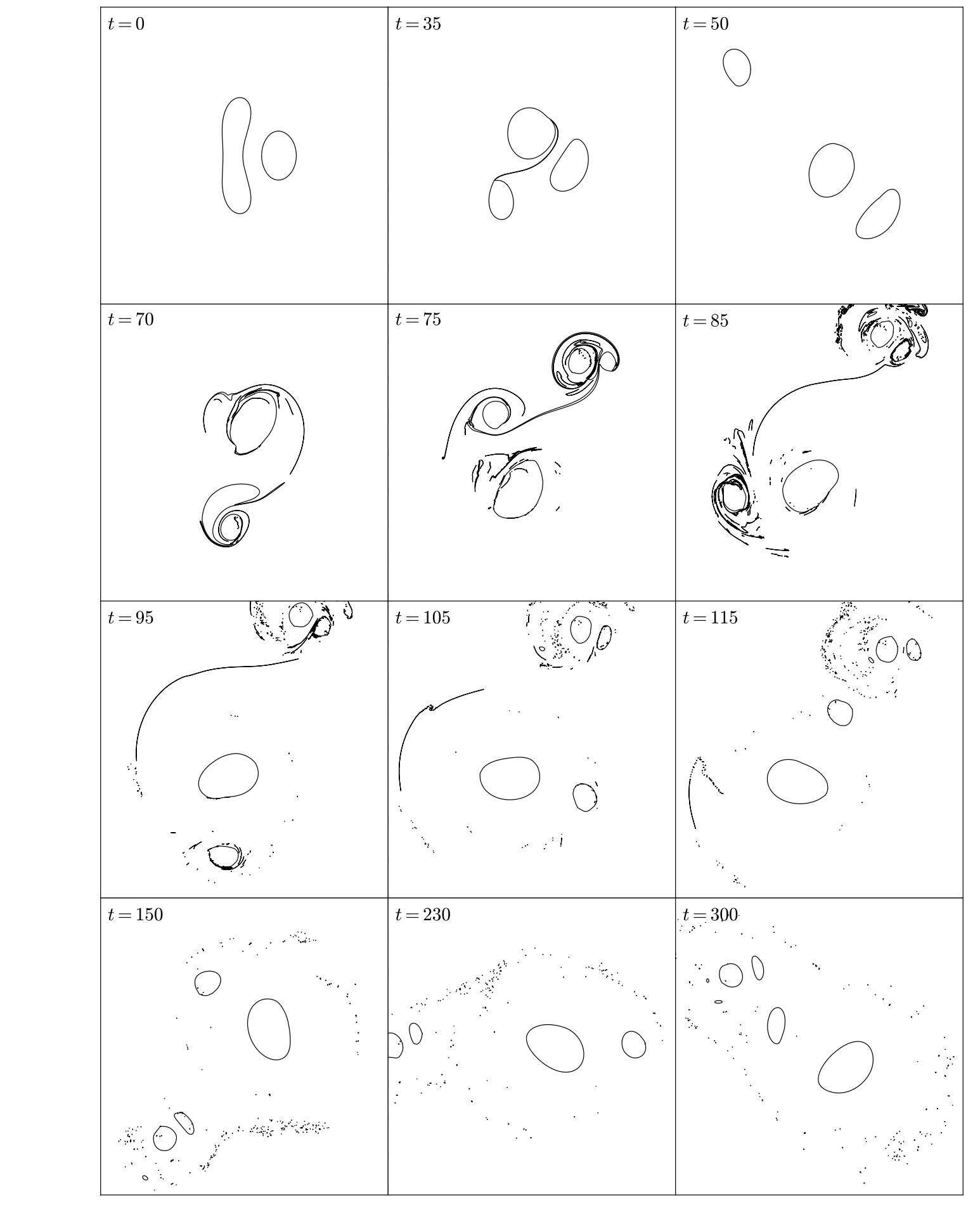

Figure 18. Case 2: nonlinear evolution from the equilibrium state having $\alpha=1 / 3, \xi=-1 / 3, \gamma=1$ and $\Omega=0.101875$.

of the larger vortex pairing up with the originally smaller vortex 2 , then exchanging with the other half. Note that the PV of vortex 1 is twice (in magnitude) that of vortex 2 , so the dipole pairs move in curved paths, taking them back towards the isolated vortex repeatedly. We could call this evolution 'exchange of partner'. This behaviour repeats itself nearly periodically until at least $t=2000$ when the simulation was discontinued. Notably, we have found many other

similar cases exhibiting this behaviour. 


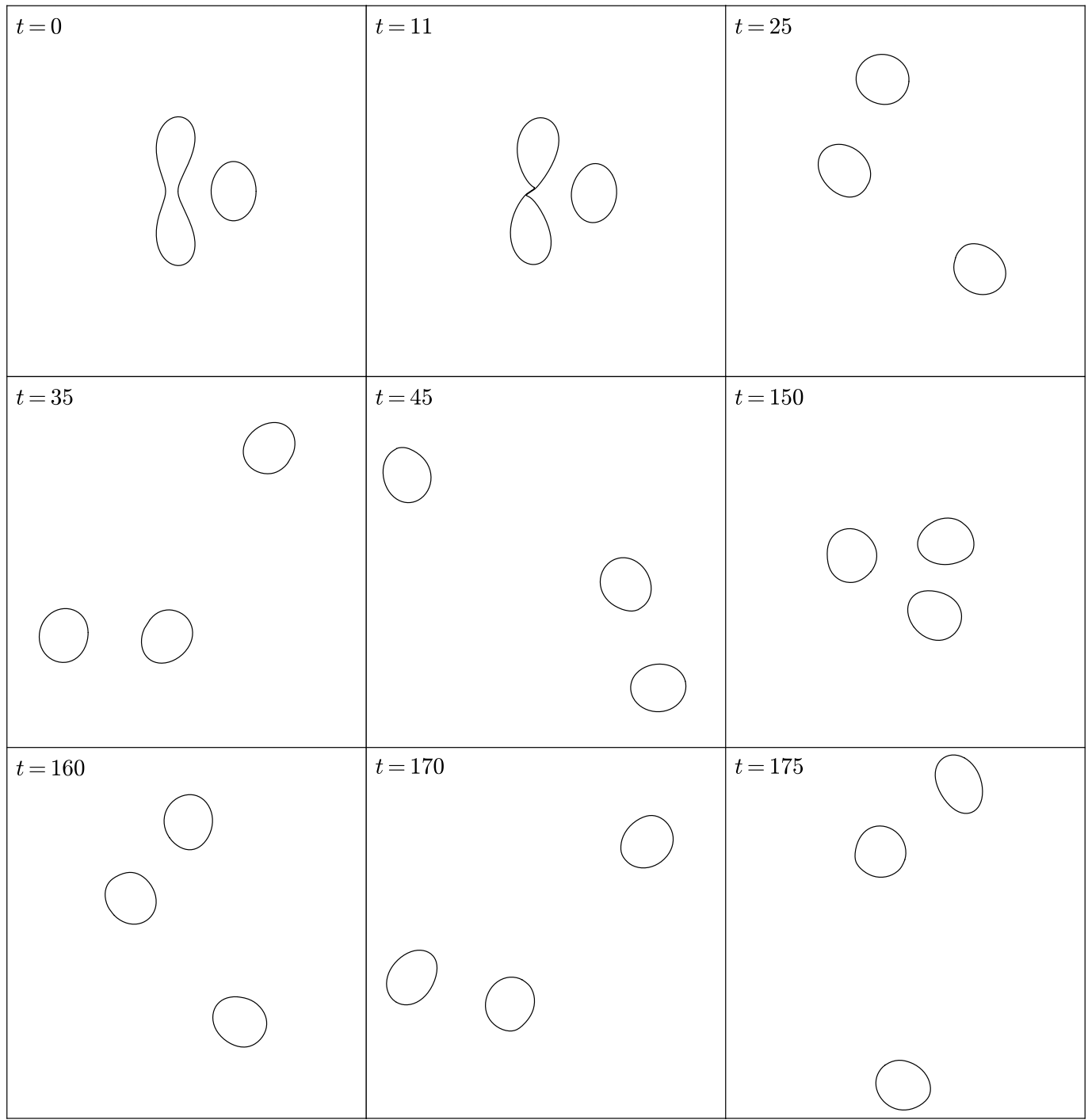

Figure 19. Case 3: nonlinear evolution from the equilibrium state having $\alpha=1 / 3, \xi=-1 / 3, \gamma=1$ and $\Omega=0.076197$.

The final case 4, near the end of the solution branch 3 in figure 16, exhibits yet another mode of linear instability with a significantly larger growth rate (see figure 4). The nonlinear evolution is shown in figure 20 (see also supplementary movie 4 ). In this case, the larger vortex does not split evenly nor cleanly. By $t=15$, vortex 2 is at the bottom of the panel, and has not experienced any strong deformation. The two parts of the original split vortex re-merge before $t=80$ then split again, only to re-merge at $t=105$ to become the large central vortex at $t=115$ and persist thereafter. The merger at $t=105$ is only partial, with a small portion of the vortex being expelled (seen to the left of the large vortex dipole visible at $t=115$ ). The original vortex 2 lies above the large central vortex at $t=115$ and is subsequently torn apart between $t=120$ and 125 by the central vortex and its weaker satellite (the top left vortex at $t=120$ ). By $t=125$, there are two main dipoles, but the smaller is separated by $t=135$ and remains loosely bound, or unbound, for the remainder of the simulation. At late times, the innermost two vortices (of significant size) orbiting around the central vortex are opposite in sign to the central vortex, while the vortex furthest away is of the same sign. This is an example of an unsteady vortex quadrupole. 


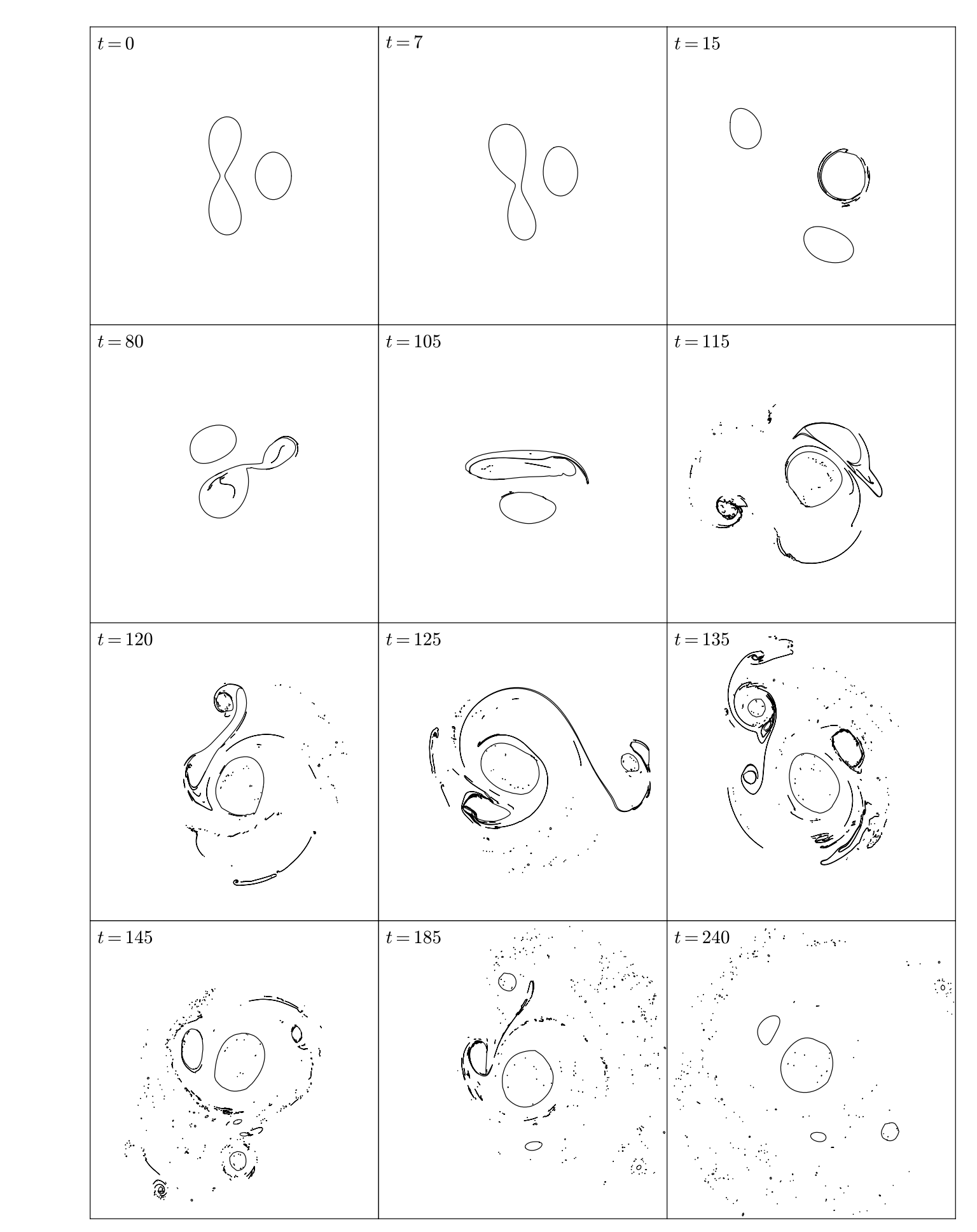

Figure 20. Case 4: nonlinear evolution from the equilibrium state having $\alpha=1 / 3, \xi=-1 / 3, \gamma=1$ and $\Omega=0.070359$.

\section{2. $\quad$ Translating vortices}

For asymmetric translating vortices, the nonlinear evolution is qualitatively similar to that seen above for rotating vortices. The primary difference is that the vortices tend to spread over a larger portion of the plane, with dipoles occasionally moving far away before returning

near the origin. Instabilities never appear to lead to perfectly matched dipoles (having equal

ner the orign. Instabilities never apper to lead to perfectly mathed diples (having equal 


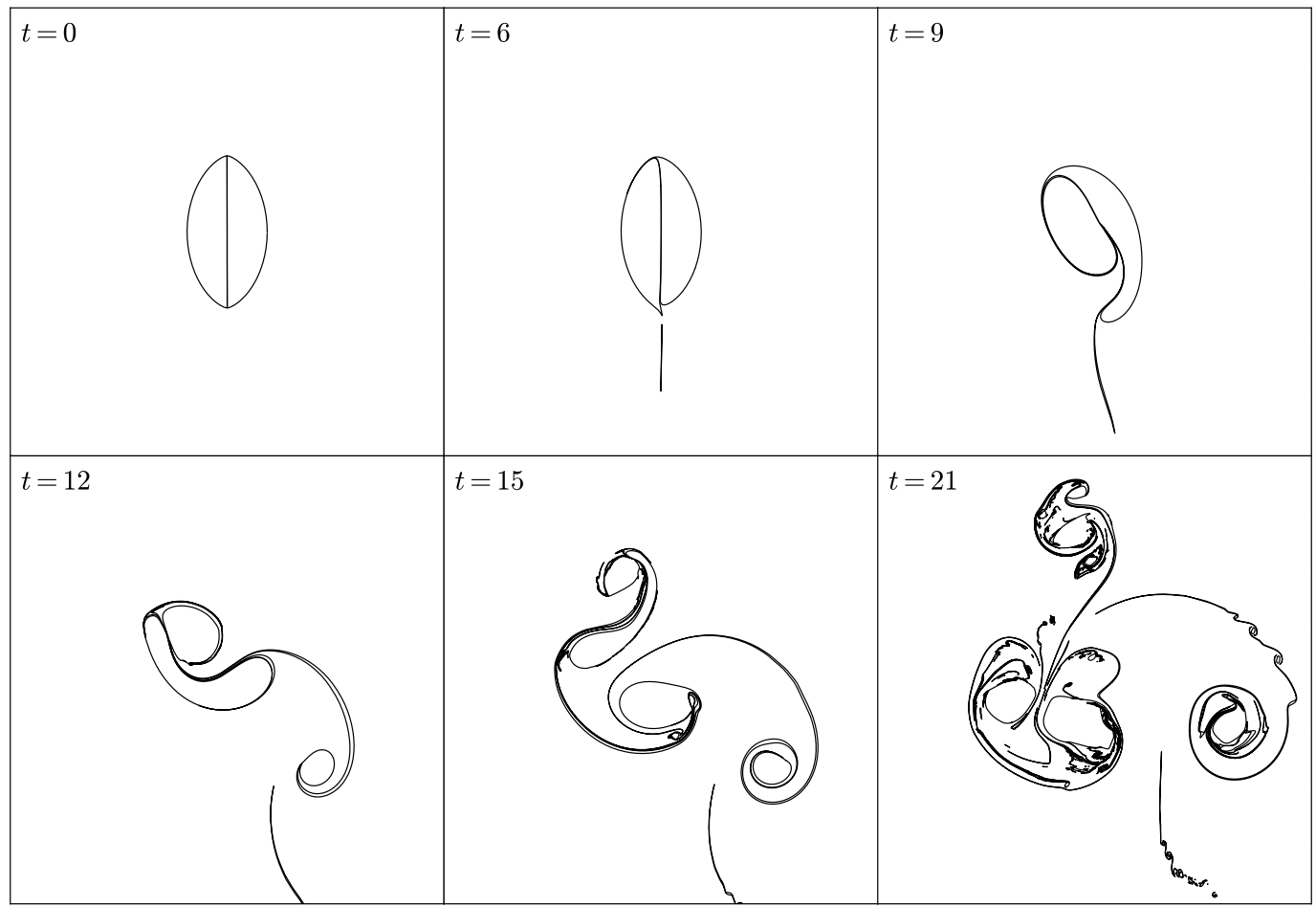

Figure 21. Nonlinear evolution of the near-limiting symmetric translating state for $\gamma=1$. Here $V=0.142152$. Note, in this and the subsequent figure, the flow is centred on a moving reference point defined by the centroid of $|q|$.

but opposite integrated PV), so dipoles invariably move on curved paths.

For symmetric translating vortices $(\alpha=1 / 2)$, any instability tends to break symmetry and leads to mismatched dipoles. However, the initial evolution can be distinctive, as shown next in two cases starting from the limiting solutions for $\gamma=1$ and 4 , respectively in figures 21 and 22 (see also supplementary movies 5 and 6). Early on, material from one or both vortices is ejected in their wake. Then one of the primary vortices deforms around the other and splits, only to subsequently deform and split the other primary vortex. This behaviour is similar to that seen in case 1 above in figure 17. As $\gamma$ increases, the amount of material ejected early on increases and symmetry is preserved for longer. The primary vortices destabilise like in the case for $\gamma=1$, and this is mirrored, in miniature, by the small dipole forming out of the ejected material. As here the material consists of two nearly symmetric opposite ribbons of PV, it forms a jet which subsequently rolls up (asymmetrically) into a vortex street. The growth in complexity here is staggering considering the relative simplicity of the initial configuration.

\subsection{Other cases}

There are too many distinctive cases to show, but some of the variations we have observed are briefly described next.

When $\gamma$ is large, here typically 4 or greater, interactions tend to produce very little filamentary debris, as previously found in Jalali and Dritschel (2018) for like-signed vortices. The exceptions are cases like that in figure 22 , where initially the close proximity of the two vortices means that there is a wide range of scales below the Rossby deformation length $L_{D}=\gamma^{-1}$. The marginally unstable symmetric translating state for $\gamma=4$ (on the same branch as the limiting case shown in figure 22) simply breaks into 3 vortices with little filamentary debris. Asymmetric limiting states in which one vortex assumes a figure of 8 shape tend to split then re-merge into an unsteady barge-like shape moving around the smaller opposite-signed vortex 


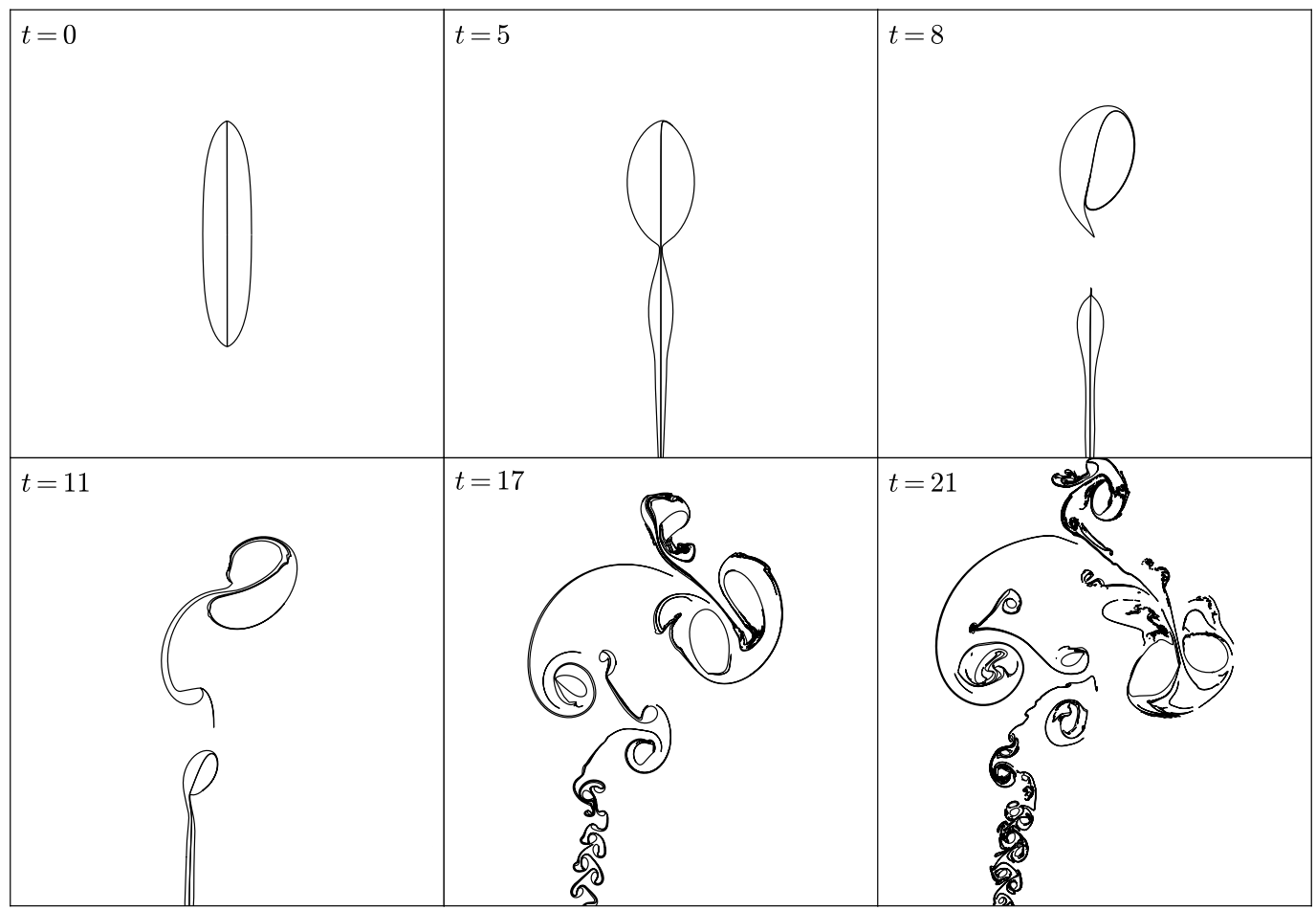

Figure 22. Nonlinear evolution of the near-limiting symmetric translating state for $\gamma=4$. Here $V=0.047510$.

(see supplementary movie 7 corresponding to $\alpha=1 / 5, \xi=-1, \gamma=4$ and $\Omega=0.004576$ ). The larger vortex may quasi-periodically enlarge at one end, and then the other. This behaviour can last for thousands of time units (perhaps indefinitely) with little or no sign of wave breaking on the vortex boundary.

In cases for which vortex 2 deforms most (typically $\alpha>1 / 2$ approximately), the first instability encountered along the branch of solutions may only lead to oscillations of vortex 2 , quasi-periodically enlarging at one end then the other, in an evidently (nonlinearly) stable manner (see supplementary movie 8 corresponding to $\alpha=3 / 5, \xi=-2 / 5, \gamma=1$ and $\Omega=$ 0.087402 ). This happens for the family of solutions illustrated in figure $5(\mathrm{a})$. The first mode of instability in figure 7 (upon decreasing $\Omega$ ) is 'exchange type' yet leads to oscillating states. This remains true until close to where the second mode of instability erupts. Then, vortex 2 splits into (in some cases) nearly equal parts, forming an unsteady tripole where the two satellites quasi-periodically move closer together then separate while remaining at nearly the same radius from the centre of the central opposite-signed vortex (see supplementary movie 9 corresponding to $\alpha=0.44, \xi=-1 / 3, \gamma=1$ and $\Omega=0.180848$ ).

\section{Conclusions}

The behaviour of two opposite-signed vortex patches in a single-layer quasi-geostrophic flow has been shown to be considerably richer than that exhibited by like-signed patches. We have computed an extensive range of families of rotating and translating (relative) equilibria, spanned by the vortex area ratio, potential vorticity ratio, Rossby deformation length and a 'branch' parameter like outer gap characterising members of a family. The latter parameter is generally difficult to choose, and often does not determine the entire family of solutions or even the family beyond its margin of stability, and it is frequently necessary to resort to a variety of parameters to compute different sections of a branch. We found that the largest 
area vortex, approximately, becomes most deformed as one approaches the limiting solution, often a figure of 8 . This is true despite the fact that this vortex may also have the largest potential vorticity. However, we have also found evidence that there are separated branches of solutions, entirely disconnected from the one stemming from two infinitely distant circular vortices, which we conjecture have limit solutions where the other vortex is most deformed. We have not attempted to compute these solutions, as there is strong evidence in a related study (Dritschel et al. 2019) that these separated branches contain only unstable solutions.

A linear stability analysis revealed that sufficiently close vortex patches are always unstable. Moreover, the margin of stability does not generally coincide with the first extremum in angular or linear impulse, for rotating and translating equilibria respectively. For like-signed vortices, this correspondence is found across the entire parameter space (Jalali and Dritschel 2018). Nonetheless, instabilities do erupt at the extremum in angular or linear impulse, and over a portion of the parameter space where one particular vortex is more deformed, this is the first instability along the branch and hence the margin of stability. Further work is necessary to understand why opposite-signed and like-signed vortices behave so differently, but we think the reason lies in the wider variety of forms of equilibria for opposite-signed vortices, evidenced by the separated solution branches.

A selected set of nonlinear simulations showed that along even a single solution branch, instabilities can evolve into a surprising variety of unsteady forms. In some cases, three main vortices are left, with one of opposite sign to the other two and lying in an inner orbit around the larger of the two remaining vortices. In other cases, four main vortices are left, and the outer two form a dipole yet orbit a central vortex, with another of opposite sign to the central vortex in a closer orbit. Yet other cases evolve into three vortices, two of which pair for a while, then one member joins the third vortex, together moving off as a pair, only to repeat the sequence. We have seen the formation of unsteady tripoles (two like-signed vortices at the same radius from a central vortex), the repeated break up and merger of vortices, the formation of highly-elongated and dynamic 'barges' (mainly for $\gamma \geq 4$ ), etc. In short, the long-term evolution is hugely diverse. It cannot be simply classified like in the case of likesigned vortices (Jalali and Dritschel 2018).

In future work, it would be worth considering the three-dimensional problem, namely the interaction of two opposite-signed volumes of uniform potential vorticity in the quasi-geostrophic model. The closest work in that of Reinaud and Dritschel (2009), who considered volumes of opposite potential vorticity (ratio -1 ), and also vortices having a height-to-mean-width aspect ratio of unity (after stretching height by the ratio of the buoyancy to Coriolis frequency). Note, the vortices can now be offset in the vertical, bringing in an additional parameter. To better understand how vortices in geophysical flows like the atmosphere and, in particular, the oceans interact and evolve into new forms, a natural next step is to extend the above study to consider general ratios of potential vorticity. The advantage of this approach is the simplicity of the quasi-geostrophic model and the use of uniform potential vorticity, thereby allowing a relatively-efficient contour-dynamics approach.

\section{References}

Adriani, A., Mura, A., Orton, G., Hansen, C., Altieri, F., Moriconi, M., Rogers, J., Eichstdt, G., Momary, T., Ingersoll, A., Filacchione, G., Sindoni, G., Tabataba-Vakili, F., Dinelli, B., Fabiano, F., Bolton, S., Connerney, J., Atreya, S., Lunine, J., Tosi, F., Migliorini, A., Grassi, D., Piccioni, G., Noschese, R., Cicchetti, A., Plainaki, C., Olivieri, A., ONeill, M., Turrini, D., Stefani, S., Sordini, R. and Amoroso, M., Cluster of cyclones encircling Jupiter's poles. Nature, 2018, 555, 216-219.

Boffetta, G., De Lillo, F. and Musacchio, S., Inverse cascade in Charney-Hasegawa-Mima turbulence. Europhys. Lett., 2002, 59, 687-693.

Burgess, B. and Dritschel, D., Long frontal waves and dynamic scaling in freely evolving equivalent barotropic flow. J. Fluid Mech., 2019, 866, R3. 
Burgess, B., Dritschel, D. and Scott, R., Extended scale invariance in the vortices of freely evolving twodimensional turbulence. Phys. Rev. Fluids, 2017, 2, 114702.

Carton, X., Oceanic Vortices. In Fronts, Waves and Vortices in Geophysical Flows, edited by J.B. Flor, Vol. 805, chap. 3, pp. 61-108, 2010 (Springer-Verlag: Berlin).

Christiansen, J. and Zabusky, N., Instability, coalescence and fission of finite-area vortex structures. J. Fluid Mech., 1973, 61, 219-243.

Deem, G. and Zabusky, N., Vortex waves: stationary V-states, interactions, recurrence, and breaking. Phys. Rev. Lett., 1978, 40, 859-862.

Dritschel, D., Contour surgery: A topological reconnection scheme for extended integrations using contour dynamics. J. Comput. Phys., 1988, 77, 240-266.

Dritschel, D., Contour dynamics and contour surgery: Numerical algorithms for extended, high-resolution modelling of vortex dynamics in two-dimensional, inviscid, incompressible flows. Computer Physics Reports, 1989, 10, 77-146.

Dritschel, D., A general theory for two-dimensional vortex interactions. J. Fluid Mech., 1995, 293, $269-303$.

Dritschel, D., Hmidi, T. and Renault, C., Imperfect Bifurcation for the Quasi-Geostrophic Shallow-Water Equations. Arch. Rational Mech. Anal., 2019, 231, 1853-1915.

Dritschel, D. and Waugh, D., Quantification of the inelastic interaction of unequal vortices in two-dimensional vortex dynamics. Phys. Fluids A, 1992, 4, 1737-1744.

Fedorov, K. and Ginsburg, A., Mushroom-like currents (vortex dipoles): one of the most widespread forms of non-stationary coherent motions in the ocean. Elsevier Oceanographic Series, 1989, 50, 1-14.

Fontane, J. and Dritschel, D., The HyperCASL Algorithm: a new approach to the numerical simulation of geophysical flows. J. Comput. Phys., 2009, 228, 6411-6425.

Jalali, M. and Dritschel, D., The interaction of two asymmetric quasi-geostrophic vortex patches. Geophys. Astrophys. Fluid Dyn., 2018, 112, 375-401.

Larichev, V. and McWilliams, J., Weakly decaying turbulence in an equivalent barotropic fluid. Phys. Fluids A, 1991, 3, 938-950.

Luzzatto-Fegiz, P. and Williamson, C., Stability of elliptical vortices from "Imperfect-Velocity-Impulse" diagrams. Theor. Comput. Fluid Dyn., 2010, 24, 181-188.

Luzzatto-Fegiz, P. and Williamson, C., An efficient and general numerical method to compute steady uniform vortices. J. Comput. Phys., 2011, 230, 6495-6511.

Makarov, V. and Kizner, Z., Stability and evolution of uniform-vorticity dipoles. J. Fluid Mech., 2011, 672, $307-325$.

Makarov, V., Sokolovskiy, M. and Kizner, Z., Doubly symmetric finite-core heton equilibria. J. Fluid Mech., 2012, 708, 397-417.

McWilliams, J., The emergence of coherent isolated vortices in turbulent flow. J. Fluid Mech., 1984, 146, 21-43.

Mied, R., McWilliams, J. and Lindemann, G., The generation and evolution of mushroom-like vortices. J. Phys. Oceanogr., 1991, 21, 489-510.

Overman II, E., Steady-state solutions of the Euler Equations in two dimensions II. Local analysis of limiting V-states. SIAM J. Appl. Math., 1986, 46, 765-800.

Płotka, H. and Dritschel, D., Quasi-geostrophic shallow-water doubly-connected vortex equilibria and their stability. J. Fluid Mech., 2013, 723, 40-68.

Polvani, L., Two-layer geostrophic vortex dynamics. Part 2. Alignment and two-layer V-states. J. Fluid Mech., 1991, 225, 241-270.

Polvani, L., Zabusky, N. and Flierl, G., Two-layer geostrophic vortex dynamics. Part 1. Upper-layer V-states and merger. J. Fluid Mech., 1989, 205, 215-242.

Reinaud, J. and Dritschel, D., Destructive interactions between two counter-rotating quasi-geostrophic vortices. J. Fluid Mech., 2009, 639, 195-211.

Vallis, G., Atmospheric and Oceanic Fluid Dynamics, 2006 (Cambridge University Press).

van Heijst, G. and Clercx, H., Laboratory modeling of geophysical vortices. Ann. Rev. Fluid Mech., 2009, 41, $143-164$.

$\mathrm{Wu}, \mathrm{H}$., Overman II, E. and Zabusky, N., Steady-state solutions of the Euler equations in two dimensions: rotating and translating V-states with limiting cases. I. Numerical algorithms and results. J. Comput. Phys., 1984, 53, 42-71.

Zabusky, N., Hughes, M. and Roberts, K., Contour Dynamics for the Euler Equations in Two Dimensions. J. Comput. Phys., 1979, 30, 96-106. 